\title{
Optimization of UAV Heading for the Ground-to-Air Uplink
}

\author{
Feng Jiang and A. Lee Swindlehurst \\ Department of Electrical Engineering and Computer Science \\ University of California, Irvine \\ Irvine, CA, 92697, USA \\ Email:\{feng.jiang, swindle\}@uci.edu
}

\begin{abstract}
In this paper we consider a collection of single-antenna ground nodes communicating with a multiantenna unmanned aerial vehicle (UAV) over a multiple-access ground-to-air wireless communications link. The UAV uses beamforming to mitigate the inter-user interference and achieve spatial division multiple access (SDMA). First, we consider a simple scenario with two static ground nodes and analytically investigate the effect of the UAV heading on the system sum rate. We then study a more general setting with multiple mobile ground-based terminals, and develop an algorithm for dynamically adjusting the UAV heading in order to maximize a lower bound on the ergodic sum rate of the uplink channel, using a Kalman filter to track the positions of the mobile ground nodes. Fairness among the users can be guaranteed through weighting the bound for each user's ergodic rate with a factor inversely proportional to their average data rate. For the common scenario where a high $K$-factor channel exists between the ground nodes and UAV, we use an asymptotic analysis to find simplified versions of the algorithm for low and high SNR. We present simulation results that demonstrate the benefits of adapting the UAV heading in order to optimize the uplink communications performance. The simulation results also show that the simplified algorithms perform near-optimal performance.
\end{abstract}

\section{Index Terms}

UAV communication networks, UAV relays, UAV positioning, interference mitigation, beamforming 


\section{INTRODUCTION}

\section{A. Background}

There is increasing interest in the use of relatively small, flexible unmanned aerial vehicles (UAVs) that fly at lower altitudes for providing relay services for mobile ad hoc networks with ground-based communication nodes [1] $[$ [8]. We consider such an application in this paper, assuming a system with a multi-antenna unmanned aerial vehicle (UAV) flying over a collection of $N$ single-antenna mobile ground nodes. The UAV acts as a decode-and-forward relay, sending the messages from the co-channel users on the ground to some remote base station. The goal is to control the motion of the UAV so as to optimize the uplink communications performance.

A number of different approaches have been proposed in the literature to address the performance of UAV-assisted communication networks. For example, in [1], a throughput maximization protocol for non-real time applications was proposed for a network with UAV relays in which the UAV first loads data from the source node and then flies to the destination node to deliver it. The authors in [2] investigated different metrics for ad hoc network connectivity and propose several approaches for improving the connectivity through deployment of a UAV. In [3], the authors considered a scenario in which multiple UAVs are deployed to relay data from isolated ground sensors to a base station, and an algorithm was proposed to maintain the connectivity of the links between the sensors and base station.

The work described above assumes that the ground nodes are static and that the UAV is configured with only a single antenna. Given the well-known benefits of employing multiple

antennas for communications, it is natural to consider the advantages they offer for UAV-based platforms [9]. The measurement results of [10] showed that using multiple receivers at the UAV can significantly increase the packet delivery rate of the ground-to-air link. A swarm of single antenna UAVs were used as a virtual antenna array to relay data from a fixed ad hoc network on the ground in [4], and the performance of distributed orthogonal space-time block codes (OSTBC) and beamforming were evaluated. A relay system with multi-antenna UAVs and multiantenna mobile ground terminals was investigated in [5]. The users employ OSTBC to transmit data and the data transmissions are assumed to be interference free. Based on estimates of the user terminals' future position, a heading optimization approach was proposed that maximizes 
the uplink sum rate of the network under the constraint that each user's rate is above a given threshold. The restriction of [5] to the interference-free case is a significant drawback, which we address in this paper. An earlier version of our work [11] discussed the use of an antenna array to improve the throughput of the ground-to-air uplink when the users share the same channel and interfere with one another.

\section{B. General Approach and Contribution}

In this paper, we consider a model similar to [5], with several ground-based users communicating simultaneously with a multi-antenna UAV. The main difference with [5] is that we assume there exists co-channel interference between the different users' data streams. The users are assumed to transmit data with a single antenna and the UAV uses beamforming to separate the co-channel data streams. We assume a correlated Rician fading channel model between each ground node and the UAV, and we use a lower bound on the ergodic achievable rate to quantify the uplink performance of the relay network, assuming that the UAV uses a maximum signalto-interference-plus-noise ratio (SINR) beamformer for interference mitigation. The strength of the mutual interference depends on the correlation between the users' channel vectors, which in a high $K$-factor channel is a function of the signals' angle of arrival (AoA). The AoAs depend in turn on the UAV's heading and the relative positions of the UAV and the ground nodes. Consequently, we propose an adaptive algorithm for adjusting the heading of the UAV to minimize the users' mutual interference and improve the uplink communications performance. In particular, the UAV is assumed to fly with a constant velocity $v_{u}$, and it adjusts its heading in discrete time steps (assuming a constraint on the maximum turn rate) in order to optimize the bound on the achievable rate. At time step $n$, the UAV uses a Kalman filter driven by feedback from the ground terminals to predict their positions at time $n+1$, and then the UAV computes its heading in order to optimize the bound based on these future position estimates.

The main results of this paper are summarized as follows:

1) We analyze the trajectory optimization problem for a special case involving two static ground nodes. We use a rectangular-path model to characterize the UAV's trajectory, which reduces the problem to one of optimizing only the heading. This problem can be solved using a simple line search, and the results indicate how increasing the size of the UAV 
array can reduce the system's sensitivity to the heading direction.

2) For the case of a general network of mobile ground-based nodes, we derive a lower bound on the average achievable sum rate to measure the system performance. Based on this lower bound, we formulate a heading optimization problem and propose a line-search algorithm to adjust the UAV's heading direction at time step $n$ such that the system performance at time step $n+1$ can be optimized. We study the performance of both time-division multiple access (TDMA) and space-division multiple access (SDMA), and illustrate via simulation the dramatic improvement offered by SDMA.

3) We derive asymptotic analytical results for the heading optimization problem under the assumption of a high $K$-factor Rician channel between the ground nodes and UAV. The asymptotic results provide simplified methods for solving the heading optimization problem. A separate approximation method is used for low and high SNR cases, and we show that using the asymptotic expressions for heading optimization results in performance nearly identical to that of the optimal algorithm.

\section{Organization}

The organization of the paper is as follows. We present our assumed signal and channel model in Section III, and in Section III we focus on the UAV heading optimization problem for the special case of two static ground users. In Section IV] we first describe the mobility model for the UAV and ground nodes, as well as a standard Kalman filter for predicting the future positions of the ground nodes. Then we formulate the UAV heading optimization problem and propose an adaptive heading adjustment algorithm. We then derive asymptotic expressions for the general heading optimization problem in Section $[\nabla$, assuming a high- $K$ factor Rician channel between the UAV and ground nodes. Simulation results are provided in Section VI to illustrate the performance of the heading control algorithm, the advantage of SDMA over TDMA, and the validity of the asymptotic results. 


\section{SySTEM MODEL}

\section{A. Signal Model}

We assume a UAV configured with an array of $M$ antennas, and a collection of $N$ ground nodes each equipped with a single antenna. We restrict attention to non-hovering UAVs that must maintain a certain forward velocity to remain airborne. We assume that, during the period

of time in which the UAV is receiving uplink data from the ground nodes, the UAV maintains a constant altitude $h_{u}$ and a constant velocity $v_{u}$. For simplicity, we assume that each ground node transmits with the same power $P_{t}$, but this assumption is easily relaxed. The signal received at the UAV array at time $n$ can thus be written as

$$
\mathbf{y}_{n}=\sum_{i=1}^{N} \sqrt{P_{t}} \mathbf{h}_{i, n} x_{i, n}+\mathbf{n}_{n},
$$

where $\mathbf{h}_{i, n} \in \mathbb{C}^{M \times 1}$ is the channel vector between node $i$ and the UAV, the data symbol $x_{i, n}$ is a complex scalar with zero mean and unit magnitude, $\mathbf{n} \in \mathbb{C}^{M \times 1}$ is zero-mean additive Gaussian noise with covariance $\mathbb{E}\left\{\mathbf{n}_{n} \mathbf{n}_{n}^{H}\right\}=\sigma^{2} \mathbf{I}_{M}$, and $\mathbf{I}_{M}$ denotes an $M \times M$ identity matrix. The UAV isolates the data from the $i$ th node by multiplying $\mathbf{y}_{n}$ with a beamformer $\mathbf{w}_{i, n}$. As such, we assume that the number of active uplink users is less than the number of antennas, or $N \leq M$. Assuming the channels $\mathbf{h}_{i, n}, i=1, \ldots, N$ are known to the UAV (e.g., via training data from the ground nodes), the vector $\mathbf{w}_{i, n}$ that maximizes the signal-to-interference-plus-noise ratio $S I N R_{i, n}$ is given by [12]

$$
\mathbf{w}_{i, n}=\mathbf{Q}_{i, n}^{-1} \mathbf{h}_{i, n}
$$

where $\mathbf{Q}_{i, n}=\sum_{j=1, j \neq i}^{N} P_{t} \mathbf{h}_{j, n} \mathbf{h}_{j, n}^{H}+\sigma^{2} \mathbf{I}_{M}$. The corresponding $S I N R_{i, n}$ can be calculated as

$$
S I N R_{i, n}=P_{t} \mathbf{h}_{i, n}^{H} \mathbf{Q}_{i, n}^{-1} \mathbf{h}_{i, n} .
$$

\section{B. Channel Model}

We assume a correlated Rician fading channel between each user node and the UAV with consideration of large-scale path loss:

$$
\mathbf{h}_{i, n}=\frac{\mathbf{h}_{i, n}^{\prime}}{d_{i, n}^{\alpha}}
$$


where $\mathbf{h}_{i, n}^{\prime}$ is the normalized channel vector, $d_{i, n}$ is the distance between node $i$ and the UAV during the $n$th time step, and $\alpha$ is the path loss exponent. Define the three dimensional coordinates of the UAV and node $i$ as $\left(x_{u, n}, y_{u, n}, h_{u}\right)$ and $\left(x_{i, n}, y_{i, n}, 0\right)$, so that $d_{i, n}$ is given by

$$
d_{i, n}=\sqrt{\left(x_{u, n}-x_{i, n}\right)^{2}+\left(y_{u, n}-y_{i, n}\right)^{2}+h_{u}^{2}}
$$

For node $i$, we write the Rician fading channel vector $\mathbf{h}_{i, n}^{\prime}$ with two components [13], a line-of-sight (LOS) component $\overline{\mathbf{h}}_{i, n}$ and a Rayleigh fading component $\tilde{\mathbf{h}}_{i, n}$ :

$$
\mathbf{h}_{i, n}^{\prime}=\overline{\mathbf{h}}_{i, n}+\tilde{\mathbf{h}}_{i, n}
$$

The LOS response will depend on the AoA of the signal, which in turn depends on the heading of the UAV (determining the orientation of the array) and the positions of the UAV and user nodes. For example, assume a uniform linear array (ULA) with antennas separated by one-half wavelength, and that at time step $n$ the phase delay between adjacent antenna elements for the signal from the $i$ th node is $p_{i, n}$, then the LOS component could be modeled as

$$
\overline{\mathbf{h}}_{i, n}=\sqrt{\frac{K}{1+K}}\left[1, e^{j p_{i, n}}, \cdots, e^{j(M-1) p_{i, n}}\right]^{T},
$$

where $K$ is the Rician $K$-factor. The phase delay $p_{i, n}$ is calculated by [14, chap. 4]

$$
p_{i, n}=\pi \cos \left(\phi_{i, n}\right) \sin \left(\theta_{i, n}\right)
$$

where $\phi_{i, n}$ and $\theta_{i, n}$ represent the elevation and azimuth angles to the $i$ th ground node. In terms of the UAV and user node positions, these quantities can be calculated as

$$
\begin{aligned}
\cos \left(\phi_{i, n}\right) & =\sqrt{\frac{\left(x_{u, n}-x_{i, n}\right)^{2}+\left(y_{u, n}-y_{i, n}\right)^{2}}{\left(x_{u, n}-x_{i, n}\right)^{2}+\left(y_{u, n}-y_{i, n}\right)^{2}+h_{u}^{2}}}, \\
\sin \left(\theta_{i, n}\right) & =\cos \left(\delta_{n}-\epsilon_{i, n}\right),
\end{aligned}
$$

where $\delta_{n}$ is the heading angle of the UAV, $\delta_{n}-\epsilon_{i, n}$ denotes the angle between the UAV heading and the LOS to user $i$, and

$$
\begin{aligned}
& \epsilon_{i, n}= \begin{cases}\zeta_{i, n}, \quad & y_{i, n}-y_{u, n} \geq 0 \text { and } x_{i, n}-x_{u, n} \geq 0 \\
\zeta_{i, n}+\pi, & x_{i, n}-x_{u, n} \leq 0 \\
\zeta_{i, n}+2 \pi, & \text { otherwise. }\end{cases} \\
& \zeta_{i, n}=\arctan \left(\frac{y_{i, n}-y_{u, n}}{x_{i, n}-x_{u, n}}\right) .
\end{aligned}
$$


Since there is little multipath scattering near the UAV, any Rayleigh fading components will experience high spatial correlation at the receive end of the link. Thus, we model the spatial correlated Rayleigh component as

$$
\tilde{\mathbf{h}}_{i, n}=\sqrt{\frac{1}{1+K}}\left(\mathbf{R}_{r}\right)^{\frac{1}{2}} \mathbf{g}_{i, n},
$$

where $\mathbf{g}_{i, n} \in \mathbb{C}^{M \times 1}$ has i.i.d. zero-mean, unit-variance complex Gaussian entries (which we denote by $\mathcal{C N}(0,1)$ ), and $\mathbf{R}_{r}$ is the spatial correlation matrix of the channel on the receiver side of the link. In [15], a model for $\mathbf{R}_{r}$ is proposed under the assumption that the multipah rays are distributed normally in two dimensions around the angle from the source with standard deviation $\sigma_{r}$, assuming a ULA receiver. We can easily extend this model to take into account the third dimension corresponding to the elevation angle, and the resulting $\mathbf{R}_{r}$ is given by

$$
\mathbf{R}_{r}=\left(1+\frac{1}{K}\right) \overline{\mathbf{h}}_{i, n} \overline{\mathbf{h}}_{i, n}^{H} \odot \mathbf{B}\left(\theta_{i, n}, \sigma_{r}\right),
$$

where $\odot$ denotes the Hadamard (element-wise) product, and

$$
\mathbf{B}\left(\theta_{i, n}, \sigma_{\phi}\right)_{k, l}=e^{-\frac{1}{4}(\pi(k-l))^{2} \sigma_{r}^{2} \cos ^{2}\left(\theta_{i, n}\right)\left(1+\cos \left(2 \phi_{i, n}\right)-\frac{1}{2} \sigma_{r}^{4} \sin ^{2}\left(2 \phi_{i, n}\right)(\pi(k-l))^{2} \cos ^{2}\left(\theta_{i, n}\right)\right)} .
$$

The resulting distribution for $\mathbf{h}_{i, n}^{\prime}$ is thus

$$
\mathbf{h}_{i, n}^{\prime} \sim \mathcal{C N}\left(\overline{\mathbf{h}}_{i, n}, \frac{1}{K+1} \mathbf{R}_{r}\right)
$$

For the remainder of the paper, we will use the channel model defined by the LOS component in (7) and the Rayleigh component in (12), which implies a UAV equipped with a ULA. The ULA could be oriented along either the fuselage or the wings of the UAV, the only difference being a $90^{\circ}$ change in how we define the heading angle. Extensions of the algorithm and analysis to different array geometries would require one to use a different expression for (7), which is straightforward, and to derive a different spatial correlation matrix $\mathbf{R}_{r}$, which is more complicated.

\section{Results for the Static Two-User CASE}

To demonstrate the significant impact of the UAV trajectory on the performance of the groundto-air uplink, we first consider a simple two user scenario. The gross behavior of the UAV would be governed by the distance $D$ between the two users, with three possibilities: 
1) $D \gg h_{u}$ - This is not a particularly useful scenario for a simultaneous uplink from both users since, if the UAV flies near their midpoint, both users would experience low SINR at the UAV due to path loss, and the sum data rate would be quite low. In this case, a better approach would likely involve the UAV serving each ground node separately, circling directly above each user and alternately flying between them.

2) $D \ll h_{u}$ - This case is also less interesting since the UAV should obviously fly directly above the two users in as tight a pattern as possible to minimize path loss. The effect of the UAV heading would be minimal, since the AoAs to the two users would be nearly identical. If the $K$-factor of the channel was high (as one would expect when the UAV is essentially directly overhead), then the channels would be highly correlated and a TDMA solution would likely be preferred over SDMA.

3) $D=O\left(h_{u}\right)$ - Since the users transmit with the same power and their channels have the same statistical properties, equalizing the average uplink rates for the two users would require the UAV to fly a symmetric trajectory centered around the midpoint of the two users. If it was desired to minimize the variation in each user's average uplink rate, the bounds of this trajectory would be small relative to the distance to the users. This is the case we consider in this section.

To make the analysis tractable, we focus on a rectangular trajectory as depicted in Fig. 1 , defined by the side lengths $C_{a}$ and $C_{b}$ and the orientation $\delta$. The angle $\delta$ is defined to be with respect to the side of the rectangle with greater length. Given the assumptions for scenario (3) above, the side lengths are assumed to satisfy $\max \left\{C_{a}, C_{b}\right\} \leq C_{\max } \ll d_{i}$, so the figure is not to scale. Under this assumption, the performance of a rectangular trajectory is expected to be similar to that for other trajectories with similar size and orientation (e.g., an ellipse or figure-8 pattern). We also assume that $\min \left\{C_{a}, C_{b}\right\} \geq C_{\min }$, which effectively accounts for the turning radius of the UAV.

The sum data rate at the UAV averaged along the trajectory is given by

$$
\begin{aligned}
\bar{R} & =\mathbb{E}\left\{\log _{2}\left(1+S I N R_{1}\right)+\log _{2}\left(1+S I N R_{2}\right)\right\} \\
& =\frac{1}{2\left(C_{a}+C_{b}\right)} \int_{\mathcal{C}}\left(\log _{2}\left(1+S I N R_{1}(p)\right)+\log _{2}\left(1+S I N R_{2}(p)\right)\right) d p
\end{aligned}
$$


where $\mathcal{C}$ denotes the rectangular path followed by the UAV, variable $p$ denotes different positions along the trajectory and $d p$ represents the length of the elementary subintervals along the trajectory. The optimization problem we wish to solve is formulated as

$$
\begin{array}{cl}
\max _{\delta, C_{a}, C_{b}} & \bar{R} \\
\text { subject to } & 0 \leq \delta \leq \frac{\pi}{2} \\
& C_{\min } \leq C_{b} \leq C_{a} \leq C_{\max }
\end{array}
$$

where the symmetry of the problem allows us to restrict attention to $0 \leq \delta \leq \pi / 2$ and assume $C_{b} \leq C_{a}$ without loss of generality. This non-linear optimization problem is difficult to solve directly. In the appendix, we show that for high SNR $\left(\frac{P_{t}}{d_{i}^{\alpha} \sigma^{2}} \gg 1\right)$ and assuming channels with a large $K$-factor, the solution to (14) is approximately given by $C_{a}=C_{\max }, C_{b}=C_{\min }$ and

$$
\delta=\arg \min _{0 \leq \delta \leq \pi / 2} \frac{R_{c}}{1+R_{c}} \frac{\sin ^{2}\left(M \pi \cos \left(\phi^{\prime}\right) \cos (\delta)\right)}{\sin ^{2}\left(\pi \cos \left(\phi^{\prime}\right) \cos (\delta)\right)}+\frac{1}{1+R_{c}} \frac{\sin ^{2}\left(M \pi \cos \left(\phi^{\prime}\right) \sin (\delta)\right)}{\sin ^{2}\left(\pi \cos \left(\phi^{\prime}\right) \sin (\delta)\right)},
$$

where $R_{c}=\frac{C_{\max }}{C_{\min }}$ and $\phi^{\prime}$ is the elevation angle to the two users at the center of the rectangle in Fig. 1, and satisfies

$$
\cos \left(\phi^{\prime}\right)=\frac{d_{i}}{\sqrt{d_{i}^{2}+h_{u}^{2}}}
$$

Minimizing (15) can be achieved by a simple line search over the interval $[0, \pi / 2]$.

To illustrate the validity of the approximate solution, we compare the average system sum rate achieved by maximizing (14) using an exhaustive search over $\left\{C_{a}, C_{b}\right\}$ for each value of $\delta$ evaluated in the approximate line search of (15). The simulation parameters were $d_{1}=d_{2}=$ $1500 \mathrm{~m}, h_{u}=350 \mathrm{~m}, C_{\min }=200 \mathrm{~m}, C_{\max }=800 \mathrm{~m}$, and $\frac{P_{t}}{\sigma^{2}}=65 \mathrm{~dB}$. The results of the simulation are plotted in Fig. 2, which shows the best rate obtained by (14) for each value of $\delta$, and the optimal value obtained from minimizing (15) for $M=2$ and $M=4$. In both cases, the approximate approach of (15) finds a trajectory orientation that yields a near-optimal uplink rate. Fig. 2 also illustrates the benefit of increasing the number of antennas at the UAV, and that proper choice of the UAV heading can have a very large impact on communications performance.

\section{Heading Optimization for a Mobile Ground Network}

In this section we consider a more general scenario in which several mobile ground nodes are present and the UAV tracks their movement. We will consider both SDMA and TDMA 
approaches. In the SDMA scheme, all of the ground nodes are transmitting simultaneously and the UAV uses beamforming for source separation. For the TDMA method, each user is allocated an equal time slot for data transmission. It is assumed that at time step $n-1$ all of the users feedback their current position to the UAV, and these data are used to predict the positions at time $n$. An adaptive heading is proposed that calculates the UAV heading at time step $n-1$ so that the network's performance at time step $n$ will be optimized.

\section{A. Mobility Model and Position Prediction}

We adopt a first-order auto-regressive (AR) model for the dynamics of the ground-based nodes [16], and we assume the nodes provide their location to the UAVs at each time step. The UAV in turn uses a Kalman filter to predict the positions of the nodes at the next time step. We define the dynamic state of user $i$ at time step $n-1$ as:

$$
\mathbf{s}_{i, n-1}=\left[x_{i, n-1}, y_{i, n-1}, v_{i, n-1}^{x}, v_{i, n-1}^{y}\right]^{T},
$$

where $v_{i, n-1}^{x}, v_{i, n-1}^{y}$ denote the velocities in the $x$ direction and $y$ direction respectively. According to the AR model, the state of node $i$ at time step $n$ is given by

$$
\begin{aligned}
\mathbf{s}_{i, n} & =\mathbf{T s}_{\mathbf{s}_{i, n-1}}+\mathbf{w}_{i, n} \\
\mathbf{T} & =\left[\begin{array}{cccc}
1 & 0 & \Delta t & 0 \\
0 & 1 & 0 & \Delta t \\
0 & 0 & 1 & 0 \\
0 & 0 & 0 & 1
\end{array}\right],
\end{aligned}
$$

where $\mathbf{w}_{i, n} \sim \mathcal{N}\left(0, \sigma_{w}^{2} \mathbf{I}_{4}\right)$ represents a process noise term. Due to the effects of delay, quantization and possible decoding errors, the UAV's knowledge of the ground nodes' position is imprecise. This effect is described by the measurement model for user $i$ 's position:

$$
\begin{aligned}
\mathbf{z}_{i, n} & =\mathbf{F} \mathbf{s}_{i, n}+\mathbf{u}_{i, n} \\
\mathbf{F} & =\left[\begin{array}{llll}
1 & 0 & 0 & 0 \\
0 & 1 & 0 & 0
\end{array}\right],
\end{aligned}
$$

where $\mathbf{u}_{i, n} \sim \mathcal{N}\left(0, \sigma_{u}^{2} \mathbf{I}_{2}\right)$ represents the observation noise. We assume a standard implementation of the Kalman filter, as follows: 
Initialization

$$
\mathbf{x}_{i, 0}=\mathbf{F s}_{i, 0}, \mathbf{P}_{i, 0}=\left[\begin{array}{ll}
0 & 0 \\
0 & 0
\end{array}\right]
$$

Prediction

$$
\begin{aligned}
\hat{\mathbf{s}}_{i, n \mid n-1} & =\mathbf{T} \hat{\mathbf{s}}_{i, n-1 \mid n-1} \\
\mathbf{P}_{i, n \mid n-1} & =\mathbf{T P}_{i, n-1 \mid n-1} \mathbf{T}+\sigma_{w}^{2} \mathbf{I}_{4}
\end{aligned}
$$

Kalman gain

$$
\mathbf{K}_{i, n}=\mathbf{P}_{i, n \mid n-1} \mathbf{F}^{T}\left(\mathbf{F P}_{i, n \mid n-1} \mathbf{F}^{T}+\sigma_{u}^{2} \mathbf{I}_{2}\right)^{-1}
$$

Measurement update

$$
\begin{aligned}
\hat{\mathbf{s}}_{i, n \mid n} & =\hat{\mathbf{s}}_{i, n \mid n-1}+\mathbf{K}_{i, n}\left(\mathbf{z}_{i, n}-\mathbf{F} \hat{\mathbf{s}}_{i, n \mid n-1}\right), \\
\mathbf{P}_{i, n \mid n} & =\left(\mathbf{I}_{4}-\mathbf{K}_{i, n} \mathbf{F}\right) \mathbf{P}_{i, n \mid n-1} .
\end{aligned}
$$

\section{B. SDMA Scenario}

The average sum rate of the uplink network can be approximated by a reasonably tight upper bound

$$
\begin{aligned}
C_{n} & =\sum_{i=1}^{N} \mathbb{E}\left\{\log _{2}\left(1+S I N R_{i, n}\right)\right\} \\
& \leq \sum_{i=1}^{N} \log _{2}\left(1+\mathbb{E}\left\{S I N R_{i, n}\right\}\right) .
\end{aligned}
$$

The UAV heading $\delta_{n}$ will impact $C_{n}$ in two ways. First, it will change the distance between the user nodes and the UAV during time step $n$, which will impact the received power. Second, and often most importantly, changes in the heading will modify the AoA of the LOS component, which impacts the ability of the beamformer to spatially separate the users. At time step $n-1$, based on the noisy observation $\mathbf{z}_{i, n-1}$, the UAV uses the Kalman filter to predict $\left(\hat{x}_{i, n}, \hat{y}_{i, n}\right)$ and hence $\mathbb{E}\left\{S I N R_{i, n}\right\}$. The heading optimization problem can thus be formulated as

$$
\begin{aligned}
& \max _{\delta_{n}} \sum_{i=1}^{N} \log _{2}\left(1+\mathbb{E}\left\{S I N R_{i, n}\right\}\right) \\
& \text { subject to }\left|\delta_{n}-\delta_{n-1}\right| \leq \Delta \delta,
\end{aligned}
$$


where $\Delta \delta$ represents that maximum change in UAV heading possible for the given time step.

The mean value of $S I N R_{i, n}$ is calculated by

$$
\begin{aligned}
\mathbb{E}\left\{S I N R_{i, n}\right\} & =\mathbb{E}\left\{P_{t} \mathbf{h}_{i, n}^{H} \mathbb{E}\left\{\mathbf{Q}_{i, n}^{-1}\right\} \mathbf{h}_{i, n}\right\} \\
& =\frac{P_{t}}{d_{i, n}^{2 \alpha}}\left(\frac{K}{K+1} \overline{\mathbf{h}}_{i, n}^{H} \mathbb{E}\left\{\mathbf{Q}_{i, n}^{-1}\right\} \overline{\mathbf{h}}_{i, n}+\frac{1}{K+1} \operatorname{tr}\left(\mathbf{R}_{r} \mathbb{E}\left\{\mathbf{Q}_{i, n}^{-1}\right\}\right)\right),
\end{aligned}
$$

where $\operatorname{tr}(\cdot)$ denotes the trace operator. Instead of working with the complicated term $\mathbb{E}\left\{\mathbf{Q}_{i, n}^{-1}\right\}$, we use instead the following lower bound based on Jensen's inequality [17, Lemma 4]:

$$
\mathbb{E}\left\{S I N R_{i, n}\right\} \geq \frac{P_{t}}{d_{i, n}^{2 \alpha}}\left(\frac{K}{K+1} \overline{\mathbf{h}}_{i, n}^{H} \mathbb{E}\left\{\mathbf{Q}_{i, n}\right\}^{-1} \overline{\mathbf{h}}_{i, n}+\frac{1}{K+1} \operatorname{tr}\left(\mathbf{R}_{r} \mathbb{E}\left\{\mathbf{Q}_{i, n}\right\}^{-1}\right)\right),
$$

where

$$
\mathbb{E}\left\{\mathbf{Q}_{i, n}\right\}=\sum_{j=1, j \neq i}^{N} \frac{P_{t}}{d_{j, n}^{2 \alpha}}\left(\frac{K}{K+1} \overline{\mathbf{h}}_{j, n} \overline{\mathbf{h}}_{j, n}^{H}+\frac{1}{K+1} \mathbf{R}_{r}\right)+\sigma^{2} \mathbf{I}_{M}
$$

We denote the lower bound on the right side of equation (30) as $\mathbb{E}_{l}\left\{S I N R_{i, n}\right\}$ and substitute it into (28), leading to a related optimization problem:

$$
\begin{aligned}
& \max _{\delta_{n}} \sum_{i=1}^{N} \log _{2}\left(1+\mathbb{E}_{l}\left\{S I N R_{i, n}\right\}\right) \\
& \text { subject to }\left|\delta_{n}-\delta_{n-1}\right| \leq \Delta \delta .
\end{aligned}
$$

Problem (31) requires finding the maximum value of a single-variable function over a fixed interval $\delta_{n} \in\left[\delta_{n-1}-\Delta \delta, \delta_{n-1}+\Delta \delta\right]$, and thus can be efficiently solved using a one-dimensional line search. Since problem (31) aims at maximizing the sum rate of the system, the algorithm may lead to a large difference in achievable rates between the users. As an alternative, we may wish to guarantee fairness among the users using, for example, the proportional fair method [18]:

$$
\begin{gathered}
\max _{\delta_{n}} \sum_{i=1}^{N} w_{i, n} \log _{2}\left(1+\mathbb{E}_{l}\left\{S I N R_{i, n}\right\}\right) \\
\text { subject to }\left|\delta_{n}-\delta_{n-1}\right| \leq \Delta \delta,
\end{gathered}
$$

where $w_{i, n} \propto \bar{R}_{i, n}$ and $\bar{R}_{i, n}$ is user $i$ 's average data rate:

$$
\bar{R}_{i, n}=\frac{1}{n-1} \sum_{k=1}^{n-1} \mathbb{E}\left\{\log _{2}\left(1+S I N R_{i, k}\right)\right\} .
$$

Based on our experience simulating the behavior of the algorithms described in (31) and (32), we propose two simple refinements that eliminate undesirable UAV behavior. First, to avoid the 
UAV frequently flying back and forth between the user nodes in an attempt to promote fairness, the weights $w_{i, n}$ in (32) are only updated every $N_{w}$ time steps rather than for every $n$. Second, we expect that the optimal position of the UAV should not stray too far from the center of gravity $(\mathrm{CoG})$ of the ground nodes. This would not be the case if the users were clustered into very widely separated groups, but such a scenario would likely warrant the UAV serving the groups individually anyway. To prevent the UAV from straying too far from the CoG, at each time step the UAV checks to see if the calculated heading would put it outside a certain range $d_{\max }$ from the CoG. If so, instead of using the calculated value, it chooses a heading that points towards the $\mathrm{CoG}$ (or as close to this heading as possible subject to the turning radius constraint). Appropriate values for $N_{w}$ and $d_{\max }$ are found empirically.

The proposed adaptive heading algorithm is summarized in the following steps:

1) Use the Kalman filter to predict the user positions $\left(\hat{x}_{i, n}, \hat{y}_{i, n}\right)$ based on the noisy observations at time step $n-1$, and construct the objective function in (31) or (32) based on the predicted positions.

2) Use a line search to find the solution of (31) or (32) for $\delta_{n} \in[0,2 \pi]$, and denote the solution as $\tilde{\delta_{n}}$. Calculate the heading interval $\mathcal{O}_{n}=\left[\delta_{n-1}-\Delta \delta, \delta_{n-1}+\Delta \delta\right]$. If $\tilde{\delta}_{n} \in \mathcal{O}_{n}$, set $\delta_{n}=\tilde{\delta_{n}}$, else set $\delta_{n}=\arg \min _{\delta}\left|\delta-\tilde{\delta_{n}}\right|$, where $\delta=\delta_{n-1}-\Delta \delta$ or $\delta_{n-1}+\Delta \delta$.

3) Check to see if the calculated heading $\delta_{n}$ will place the UAV at a distance of $d_{\max }$ or greater from the predicted CoG of the users. If so, set $\delta_{n}=\delta_{g}$, where $\delta_{g}$ is the heading angle corresponding to the $\mathrm{CoG}$, or set $\delta_{n}=\arg \min _{\delta}\left|\delta-\delta_{g}\right|$, where $\delta=\delta_{n-1}-\Delta \delta$ or $\delta_{n-1}+\Delta \delta$.

4) UAV flies with heading $\delta_{n}$ during time step $n$.

Note that the line search in step 2 is over $[0,2 \pi]$ rather than just $\left[\delta_{n-1}-\Delta \delta, \delta_{n-1}+\Delta \delta\right]$, and the boundary point closest to the unconstrained maximum is chosen rather than the boundary with the maximum predicted rate. Thus, the algorithm may temporarily choose a lower overall rate in pursuit of the global optimum, although this scenario is uncommon. 


\section{TDMA Scenario}

In the TDMA scenario, each node is assigned one time slot for data transmission. After maximum ratio combining at the receiver, the signal-to-noise ratio (SNR) of user $i$ is given by

$$
S N R_{i, n}=\frac{P_{t}}{\sigma^{2}}\left\|\mathbf{h}_{i, n}\right\|^{2}
$$

whose mean can be calculated as

$$
\mathbb{E}\left\{S N R_{i, n}\right\}=\frac{P_{t} M}{d_{i, n}^{2 \alpha} \sigma^{2}} .
$$

For the TDMA scenario, the optimization problem is formulated as

$$
\begin{gathered}
\max _{\delta_{n}} \frac{1}{N} \sum_{i=1}^{N} w_{i, n} \log _{2}\left(1+\frac{P_{t} M}{d_{i, n}^{2 \alpha} \sigma^{2}}\right) \\
\text { subject to }\left|\delta_{n}-\delta_{n-1}\right| \leq \Delta \delta .
\end{gathered}
$$

where

$$
w_{i, n}= \begin{cases}1 & \text { max sum rate }, \\ \propto \bar{R}_{i} & \text { proportional fair. }\end{cases}
$$

The objective function in (35) can be substituted in step 2 of the adaptive heading algorithm to implement the TDMA approach.

\section{Asymptotically Approximate Heading Algorithms}

Under certain conditions, we can eliminate the need for the bound in (30) when defining our adaptive heading control algorithm and simplify the algorithm implementation. In this section, we explore the asymptotic form of $S I N R_{i, n}$ under both low and high SNR conditions. We show that in the low-SNR case, the optimal heading can be found in closed-form, without the need for a line search. In the high-SNR case, we show that maximizing the sum rate is equivalent to minimizing the sum of the users channel correlations, which can be achieved by checking a finite set of candidate headings. In Section VI, we show that the simpler asymptotic algorithms derived here provide performance essentially identical to the line-search algorithm of the previous section. Our discussion here will focus on the max-sum-rate case for SDMA; extensions to the proportional fair and TDMA cases are straightforward. 


\section{A. Asymptotic Analysis for Low SNR Case}

For low SNR $\frac{P_{t}}{d_{i, n}^{2 \alpha} \sigma^{2}} \ll 1$, the average sum rate in (27) is approximated by

$$
C_{n} \approx \sum_{i}^{N} \mathbb{E}\left\{S I N R_{i, n}\right\}
$$

and problem (31) can be rewritten as follows

$$
\begin{array}{r}
\max _{\delta_{n}} \sum_{i}^{N} \mathbb{E}\left\{S I N R_{i, n}\right\} \\
\text { subject to }\left|\delta_{n}-\delta_{n-1}\right| \leq \Delta \delta .
\end{array}
$$

In this case we can approximate $\mathrm{Q}_{i, n}^{-1}$ with the first order Neumann series [19, Theorem 4.20]:

$$
\mathbf{Q}_{i, n}^{-1} \approx \frac{1}{\sigma^{2}}\left(\mathbf{I}_{M}-\sum_{j=1, j \neq i}^{N} \frac{P_{t}}{\sigma^{2}} \mathbf{h}_{j, n} \mathbf{h}_{j, n}^{H}\right) .
$$

Substituting (38) into (3), the $S I N R_{i, n}$ for low SNR can be further expressed as

$$
S I N R_{i, n}=\frac{P_{t}}{\sigma^{2}} \mathbf{h}_{i, n}^{H}\left(\mathbf{I}_{M}-\sum_{j=1, j \neq i}^{N} \frac{P_{t}}{\sigma^{2}} \mathbf{h}_{j, n} \mathbf{h}_{j, n}^{H}\right) \mathbf{h}_{i, n},
$$

and we have

$$
\begin{aligned}
\mathbb{E}\left\{S I N R_{i, n}\right\}= & \mathbb{E}\left\{\frac{P_{t}}{\sigma^{2}} \mathbf{h}_{i, n}^{H}\left(\mathbf{I}_{M}-\sum_{j=1, j \neq i}^{N} \frac{P_{t}}{\sigma^{2}} \mathbf{h}_{j, n} \mathbf{h}_{j, n}^{H}\right) \mathbf{h}_{i, n}\right\} \\
= & \frac{P_{t}}{d_{i, n}^{2 \alpha} \sigma^{2}}\left(\frac{K}{K+1} \overline{\mathbf{h}}_{i, n}^{H}\left(\mathbf{I}_{M}-\sum_{j=1, j \neq i}^{N} \frac{P_{t}}{d_{j, n}^{2 \alpha} \sigma^{2}}\left(\frac{K}{K+1} \overline{\mathbf{h}}_{j, n} \overline{\mathbf{h}}_{j, n}^{H}+\frac{1}{K+1} \mathbf{R}_{r}\right)\right) \overline{\mathbf{h}}_{i, n}\right. \\
& \left.+\frac{1}{K+1} \operatorname{tr}\left(\mathbf{R}_{r}-\sum_{j=1, j \neq i}^{N} \frac{P_{t}}{d_{j, n}^{2 \alpha} \sigma^{2}}\left(\frac{K}{K+1} \mathbf{R}_{r} \overline{\mathbf{h}}_{j, n} \overline{\mathbf{h}}_{j, n}^{H}+\frac{1}{K+1} \mathbf{R}_{r}^{2}\right)\right)\right) \\
& \stackrel{(a)}{\approx} \frac{P_{t}}{d_{i, n}^{2 \alpha} \sigma^{2}}\left(M-\sum_{j=1, j \neq i}^{N} \frac{P_{t}}{d_{j, n}^{2 \alpha} \sigma^{2}}\left|\overline{\mathbf{h}}_{i, n}^{H} \overline{\mathbf{h}}_{j, n}\right|^{2}\right)
\end{aligned}
$$

where $(a)$ is based on the assumption of a large Rician factor $K$ for the ground-to-air channel. When scaled by $\frac{P_{t}}{d_{i, n}^{2 \alpha} \sigma^{2}} \ll 1$, the term involving $\left|\overline{\mathbf{h}}_{i, n}^{H} \overline{\mathbf{h}}_{j, n}\right|^{2}$ in the above equation plays a minor role in determining the value of $\mathbb{E}\left\{S I N R_{i, n}\right\}$. Assuming $\Delta \delta$ and the ratio $\frac{v}{d_{i, n}}$ are small enough, we 
treat $\left|\overline{\mathbf{h}}_{i, n}^{H} \overline{\mathbf{h}}_{j, n}\right|$ as a constant when $\delta_{n}$ varies in $\left[\delta_{n-1}-\Delta \delta, \delta_{n-1}+\Delta \delta\right]$. We approximate $\left|\overline{\mathbf{h}}_{i, n}^{H} \overline{\mathbf{h}}_{j, n}\right|$ as

$$
\left|\overline{\mathbf{h}}_{i, n}^{H} \overline{\mathbf{h}}_{j, n}\right| \approx\left|\overline{\mathbf{h}}_{i, n}^{\prime H} \overline{\mathbf{h}}_{j, n}^{\prime}\right|=\left|\frac{\sin \left(\frac{M \pi}{2}\left(\cos \left(\phi_{i, n}^{\prime}\right) \cos \left(\delta_{n-1}-\epsilon_{i, n}^{\prime}\right)-\cos \left(\phi_{j, n}^{\prime}\right) \cos \left(\delta_{n-1}-\epsilon_{j, n}^{\prime}\right)\right)\right)}{\sin \left(\frac{\pi}{2}\left(\cos \left(\phi_{i, n}^{\prime}\right) \cos \left(\delta_{n-1}-\epsilon_{i, n}^{\prime}\right)-\cos \left(\phi_{j, n}^{\prime}\right) \cos \left(\delta_{n-1}-\epsilon_{j, n}^{\prime}\right)\right)\right)}\right|,
$$

where $\phi_{i, n}^{\prime}$ and $\epsilon_{i, n}^{\prime}$ are calculated assuming the user nodes are located at $\left(\hat{x}_{i, n}, \hat{y}_{i, n}\right)$ and the UAV is at $\left(x_{u, n-1}, y_{u, n-1}, h_{u}\right)$ with heading $\delta_{n-1}$. The idea here is to use the UAV's position at time step $n-1$ to calculate the users' AoA at time step $n$. Moreover, $\frac{1}{d_{i, n}^{2 \alpha}}$ can be approximated in the following way

$$
\begin{aligned}
\frac{1}{d_{i, n}^{2 \alpha}}= & \left(\left(x_{u, n-1}+v \cos \delta_{n}-x_{i, n}\right)^{2}+\left(y_{u, n-1}+v \sin \delta_{n}-y_{i, n}\right)^{2}+h_{r}^{2}\right)^{-\alpha} \\
= & \left(\left(x_{u, n-1}-x_{i, n}\right)^{2}+\left(y_{u, n-1}-y_{i, n}\right)^{2}+v^{2}+h r^{2}+2\left(x_{u, n-1}-x_{i, n}\right) v \cos \left(\delta_{n}\right)\right. \\
& \left.+2\left(y_{u, n-1}-y_{i, n}\right) v \sin \left(\delta_{n}\right)\right)^{-\alpha} \\
\approx & a_{i, n}-b_{i, n} \cos \left(\delta_{n}\right)-c_{i, n} \sin \left(\delta_{n}\right),
\end{aligned}
$$

where $a_{i, n}, b_{i, n}$ and $c_{i, n}$ are defined as follows

$$
\begin{aligned}
& a_{i, n}=\left(\left(x_{u, n-1}-x_{i, n}\right)^{2}+\left(y_{u, n-1}-y_{i, n}\right)^{2}+v^{2}+h r^{2}\right)^{-\alpha} \\
& b_{i, n}=2 \alpha v\left(x_{u, n-1}-x_{i, n}\right)\left(\left(x_{u, n-1}-x_{i, n}\right)^{2}+\left(y_{u, n-1}-y_{i, n}\right)^{2}+v^{2}+h r^{2}\right)^{-(\alpha+1)} \\
& c_{i, n}=2 \alpha v\left(y_{u, n-1}-y_{i, n}\right)\left(\left(x_{u, n-1}-x_{i, n}\right)^{2}+\left(y_{u, n-1}-y_{i, n}\right)^{2}+v^{2}+h r^{2}\right)^{-(\alpha+1)} .
\end{aligned}
$$

Substituting (41) and (42) into (40), $C_{n}$ can be approximated as

$$
\begin{aligned}
C_{n} \approx & \frac{P_{t}}{\sigma^{2}} \sum_{i=1}^{N} M\left(a_{i, n}-b_{i, n} \cos \left(\delta_{n}\right)-c_{i, n} \sin \left(\delta_{n}\right)\right)-\left(\frac{P_{t}}{\sigma^{2}}\right)^{2} \sum_{i=1}^{N} \sum_{j=1, j \neq i}^{N}\left|\overline{\mathbf{h}}_{i, n}^{\prime H} \overline{\mathbf{h}}_{j, n}^{\prime}\right|^{2}\left(a_{i, n} a_{j, n}\right. \\
& \left.\quad-\left(a_{i, n} b_{j, n}+b_{i, n} a_{j, n}\right) \cos \left(\delta_{n}\right)-\left(a_{i, n} c_{j, n}+c_{i, n} a_{j, n}\right) \sin \left(\delta_{n}\right)\right) \\
= & \frac{M P_{t}}{\sigma^{2}} \sum_{i=1}^{N} a_{i, n}-\left(\frac{P_{t}}{\sigma^{2}}\right)^{2} \sum_{i=1}^{N} \sum_{j=1, j \neq i}^{N}\left|\overline{\mathbf{h}}_{i, n}^{\prime H} \overline{\mathbf{h}}_{j, n}^{\prime}\right|^{2} a_{i, n} a_{j, n}-\left(\frac{M P_{t}}{\sigma^{2}} \sum_{i=1}^{N} b_{i, n}\right. \\
& \left.-\left(\frac{P_{t}}{\sigma^{2}}\right)^{2} \sum_{i=1}^{N} \sum_{j=1, j \neq i}^{N}\left|\overline{\mathbf{h}}_{i, n}^{\prime} \overline{\mathbf{h}}_{j, n}^{\prime}\right|^{2}\left(a_{i, n} b_{j, n}+b_{i, n} a_{j, n}\right)\right) \cos \left(\delta_{n}\right)-\left(\frac{M P_{t}}{\sigma^{2}} \sum_{i=1}^{N} c_{i, n}\right. \\
& \left.-\left(\frac{P_{t}}{\sigma^{2}}\right)^{2} \sum_{i=1}^{N} \sum_{j=1, j \neq i}^{N}\left|\overline{\mathbf{h}}_{i, n}^{\prime}{ }_{i, n}^{\prime} \overline{\mathbf{h}}_{j, n}^{\prime}\right|^{2}\left(a_{i, n} c_{j, n}+c_{i, n} a_{j, n}\right)\right) \sin \left(\delta_{n}\right) .
\end{aligned}
$$


Define the first two terms in (43) as $A_{n}$, and the term multiplying $\cos \left(\delta_{n}\right)$ and $\sin \left(\delta_{n}\right)$ as $B_{n}$ and $D_{n}$, respectively. Then (43) can be further expressed as

$$
C_{n}=A_{n}-\sqrt{B_{n}^{2}+D_{n}^{2}} \cos \left(\delta_{n}-\psi_{n}\right)
$$

where

$$
\psi_{n}= \begin{cases}\arctan \left(\frac{D_{n}}{B_{n}}\right) \quad \text { if } B_{n} \geq 0 \\ \arctan \left(\frac{D_{n}}{B_{n}}\right)+\pi \quad \text { otherwise. }\end{cases}
$$

From this expression, we see that the average sum rate $C_{n}$ can be written as a sinusoidal function of $\delta_{n}$, and the maximizing heading $\delta_{n}$ is given by

$$
\delta_{n}^{*}=\bmod _{2 \pi}\left(\psi_{n}+\pi\right)
$$

As a result, for low-SNR, the following closed-form approximation to problem (37) can be used:

$$
\delta_{n}=\left\{\begin{array}{l}
\delta_{n}^{*} \delta_{n-1}-\Delta \delta<\delta_{n}^{*}<\delta_{n-1}+\Delta \delta \\
\delta_{n-1}-\Delta \delta \bmod _{\pi}\left(\left|\delta_{n-1}-\Delta \delta-\delta_{n}^{*}\right|\right)<\bmod _{\pi}\left(\left|\delta_{n-1}+\Delta \delta-\delta_{n}^{*}\right|\right) \\
\delta_{n-1}+\Delta \delta \bmod _{\pi}\left(\left|\delta_{n-1}-\Delta \delta-\delta_{n}^{*}\right|\right) \geq \bmod _{\pi}\left(\left|\delta_{n-1}+\Delta \delta-\delta_{n}^{*}\right|\right) .
\end{array}\right.
$$

\section{B. Asymptotic Analysis for High SNR Case}

In the high SNR case where $\frac{P_{t}}{d_{i, n}^{2 \alpha} \sigma^{2}} \gg 1$, the average sum rate maximization problem can be approximated as

$$
\begin{array}{r}
\max _{\delta_{n}} \prod_{i=1}^{N} \mathbb{E}\left\{S I N R_{i, n}\right\} \\
\text { subject to }\left|\delta_{n}-\delta_{n-1}\right| \leq \Delta \delta .
\end{array}
$$

Here, when $\frac{P_{t}}{d_{i, n}^{2 \alpha} \sigma^{2}} \gg 1$, we approximate $\mathbf{Q}_{i, n}^{-1}$ as follows:

$$
\begin{aligned}
\mathbf{Q}_{i, n}^{-1} & =\frac{1}{\sigma^{2}}\left(\mathbf{I}_{M}+\frac{P_{t}}{\sigma^{2}} \mathbf{H}_{i, n} \mathbf{D}_{i, n} \mathbf{H}_{i, n}^{H}\right)^{-1} \\
& \stackrel{(b)}{=} \frac{1}{\sigma^{2}}\left(\mathbf{I}_{M}-\frac{P_{t}}{\sigma^{2}} \mathbf{H}_{i, n} \mathbf{D}_{i, n}\left(\mathbf{I}_{M}+\frac{P_{t}}{\sigma^{2}} \mathbf{H}_{i, n}^{H} \mathbf{H}_{i, n} \mathbf{D}_{i, n}\right)^{-1} \mathbf{H}_{i, n}^{H}\right) \\
& \stackrel{(c)}{\approx} \frac{1}{\sigma^{2}}\left(\mathbf{I}_{M}-\mathbf{H}_{i, n}\left(\mathbf{H}_{i, n}^{H} \mathbf{H}_{i, n}\right)^{-1} \mathbf{H}_{i, n}^{H}\right),
\end{aligned}
$$


where (b) is due to the matrix inversion lemma, (c) is due to the approximation $\left(\mathbf{I}_{M}+\frac{P_{t}}{\sigma^{2}} \mathbf{H}_{i, n}^{H} \mathbf{H}_{i, n} \mathbf{D}_{i, n}\right)^{-1} \approx$ $\left(\frac{P_{t}}{\sigma^{2}} \mathbf{H}_{i, n}^{H} \mathbf{H}_{i, n} \mathbf{D}_{i, n}\right)^{-1}$, and

$$
\begin{aligned}
& \mathbf{D}_{i, n}=\operatorname{diag}\left\{\frac{1}{d_{1, n}^{2 \alpha}}, \cdots, \frac{1}{d_{i-1, n}^{2 \alpha}}, \frac{1}{d_{i+1, n}^{2 \alpha}}, \cdots, \frac{1}{d_{N, n}^{2 \alpha}}\right\} \\
& \mathbf{H}_{i, n}=\left[\mathbf{h}_{1, n} \cdots \mathbf{h}_{i-1, n} \mathbf{h}_{i+1, n} \cdots \mathbf{h}_{N, n}\right]
\end{aligned}
$$

are formed by eliminating the terms for user $i$. Plugging (48) into (3), we obtain

$$
S I N R_{i, n} \approx \frac{P_{t}}{\sigma^{2} d_{i, n}^{2 \alpha}}\left(\mathbf{h}_{i, n}^{H} \mathbf{h}_{i, n}-\left\|\mathbf{h}_{i, n}^{H} \mathbf{H}_{i, n}\left(\mathbf{H}_{i, n}^{H} \mathbf{H}_{i, n}\right)^{-1} \mathbf{H}_{i, n}^{H}\right\|^{2}\right)
$$

For large $K$-factor channels we ignore the contribution of the Rayleigh term, so that

$$
\mathbb{E}\left\{S I N R_{i, n}\right\} \approx \frac{P_{t}}{\sigma^{2} d_{i, n}^{2 \alpha}}\left(M-\left\|\overline{\mathbf{h}}_{i, n}^{H} \overline{\mathbf{H}}_{i, n}\left(\overline{\mathbf{H}}_{i, n}^{H} \overline{\mathbf{H}}_{i, n}\right)^{-1} \overline{\mathbf{H}}_{i, n}^{H}\right\|^{2}\right),
$$

where $\overline{\mathbf{H}}_{i, n}$ is defined similarly to $\mathbf{H}_{i, n}$. Thus, the heading optimization problem can be written as

$$
\begin{aligned}
& \max _{\delta_{n}} \prod_{i=1}^{N} \frac{P_{t}}{d_{i, n}^{\alpha} \sigma^{2}} \prod_{i=1}^{N}\left(M-\left\|\overline{\mathbf{h}}_{i, n}^{H} \overline{\mathbf{H}}_{i, n}\left(\overline{\mathbf{H}}_{i, n}^{H} \overline{\mathbf{H}}_{i, n}\right)^{-1} \overline{\mathbf{H}}_{i, n}^{H}\right\|^{2}\right) \\
& \text { subject to }\left|\delta_{n}-\delta_{n-1}\right| \leq \Delta \delta .
\end{aligned}
$$

At this point we make two further approximations. First, we will ignore the terms in the product involving $1 / d_{i, n}$, since $d_{i, n}$ will not change appreciably over one time step compared with the terms involving products of $\overline{\mathbf{h}}_{i, n}$, which are angle-dependent. Second, we will make the assumption that the matrix $\overline{\mathbf{H}}_{i, n}^{H} \overline{\mathbf{H}}_{i, n}$ is approximately diagonal, which implies that the UAV attempts to orient itself so that the correlation between the mean channel vectors for different users is minimized. If we then apply these two assumptions to (51), we find that the heading problem reduces to

$$
\begin{array}{r}
\min _{\delta_{n}} \sum_{i=1}^{N} \sum_{j=i+1}^{N}\left|\overline{\mathbf{h}}_{i, n}^{H} \overline{\mathbf{h}}_{j, n}\right| \\
\text { subject to }\left|\delta_{n}-\delta_{n-1}\right| \leq \Delta \delta,
\end{array}
$$

which is consistent with the assumption of minimizing inter-user channel correlation.

In Fig. 3, we show a plot of $\left|\overline{\mathbf{h}}_{i, n}^{H} \overline{\mathbf{h}}_{j, n}\right|$ for $M=4$ as a function of the difference in AoA between the two users (variable $x$ in the plot). It is clear that $\left|\overline{\mathbf{h}}_{i, n}^{H} \overline{\mathbf{h}}_{j, n}\right|$ is a piecewise concave 
function. Since a sum of concave functions is also concave, the criterion in (52) is piecewise concave as well. Since the minimum of a concave function must be located at the boundary of its domain, to find the solution to (52) it is enough to evaluate the criterion at the boundary points $\left\{\delta_{n-1}-\Delta \delta, \delta_{n-1}+\Delta \delta\right\}$ and the zero points of $\left|\overline{\mathbf{h}}_{i, n}^{H} \overline{\mathbf{h}}_{j, n}\right|$ located within $\left[\delta_{n-1}-\Delta \delta, \delta_{n-1}+\Delta \delta\right]$.

To find the zero locations, we use the fact that a piecewise quadratic approximation to $\left|\overline{\mathbf{h}}_{i, n}^{H} \overline{\mathbf{h}}_{j, n}\right|$ is very accurate (as depicted in Fig. 2). When $\Delta \delta$ is not too large, the phase term $p_{i, n}$ in (7) satisfies

$$
p_{i, n} \approx \pi \cos \left(\phi_{i, n}^{\prime}\right)\left(\cos \left(\epsilon_{i, n}^{\prime}-\delta_{n-1}\right)+\sin \left(\epsilon_{i, n}^{\prime}-\delta_{n-1}\right)\left(\delta_{n}-\delta_{n-1}\right)\right)=e_{i, n}+f_{i, n} x,
$$

where $x=\delta_{n}-\delta_{n-1}, e_{i, n}=\pi \cos \left(\phi_{i, n}^{\prime}\right) \cos \left(\epsilon_{i, n}^{\prime}-\delta_{n-1}\right), f_{i, n}=\pi \cos \left(\phi_{i, n}^{\prime}\right) \sin \left(\epsilon_{i, n}^{\prime}-\delta_{n-1}\right)$, $x \in[-\Delta \delta, \Delta \delta]$ and the calculation of $\phi_{i, n}^{\prime}$ and $\epsilon_{i, n}^{\prime}$ follows (41). Based on (53), we obtain

$$
\left|\overline{\mathbf{h}}_{i, n}^{H} \overline{\mathbf{h}}_{j, n}\right| \approx\left|\frac{\sin \left(\frac{M}{2}\left(\left(f_{i, n}-f_{j, n}\right) x+e_{i, n}-e_{j, n}\right)\right)}{\sin \left(\frac{1}{2}\left(\left(f_{i, n}-f_{j, n}\right) x+e_{i, n}-e_{j, n}\right)\right)}\right|
$$

Then the zero points of $\left|\overline{\mathbf{h}}_{i, n}^{H} \overline{\mathbf{h}}_{j, n}\right|$ in terms of $x$ are approximately given by 1

$$
z_{k}^{i, j}=\frac{2 k \pi / M-e_{i, n}+e_{j, n}}{f_{i, n}-f_{j, n}}, \quad k= \pm 1, \ldots, \pm 2 M-1 .
$$

Finally, the asymptotic solution to problem (52) can be written as

$$
\delta_{n}=\arg \min _{\delta_{n}} \sum_{i=1}^{N} \sum_{j=i+1}^{N}\left|\overline{\mathbf{h}}_{i, n}^{H} \overline{\mathbf{h}}_{j, n}\right|, \delta_{n} \in\left\{z_{k}^{i, j} \in[-\Delta \delta, \Delta \delta]\right\} \cup\left\{\delta_{n-1}-\Delta \delta, \delta_{n-1}+\Delta \delta\right\} .
$$

\section{Simulation Results}

A simulation example involving a UAV with a 4-element ULA and four user nodes was carried out to test the performance of the proposed algorithm. The time between UAV heading updates was set to $\Delta t=1 \mathrm{~s}$, and the simulation was conducted over $L=300$ steps. In the simulation, we assume the users have the same initial velocity, and then move independently according to the model described earlier. The initial velocity of the nodes is $10 \mathrm{~m} / \mathrm{s}$, and their initial positions in meters are $(0,25),(240,20),(610,30),(1240,20)$. The elements of the process and measurement noise vectors are assumed to be independent with variances given by $\sigma_{w}^{2}=0.5$ and $\sigma_{u}^{2}=0.1$,

${ }^{1}$ Where we assume $\Delta \delta<1,\left|\left(f_{i, n}-f_{j, n}\right) x+e_{i, n}-e_{j, n}\right|<4 \pi$ and we only consider the zero points in $[-4 \pi, 4 \pi]$. 
respectively. The user's transmit power is set to $\frac{P_{t}}{\sigma^{2}}=45 \mathrm{~dB}$ and the path loss exponent is $\alpha=1$. When $L=150$, all the nodes make a sharp turn and change their velocity according to $v_{i, 150}^{y} / v_{i, 150}^{x}=-1.8856$. The initial position of the UAV is $\left(x_{u, 0}, y_{u, 0}\right)=(50,100) \mathrm{m}$ and its altitude is assumed to be $h_{u}=350 \mathrm{~m}$. The speed of the UAV is $v_{u}=50 \mathrm{~m} / \mathrm{s}$, and the maximum heading angle change is set to be either $\Delta \delta=\frac{\pi}{6}$ or $\frac{\pi}{9}$ depending on the case considered. The angle spread factor in (11) is set to $\sigma_{r}^{2}=0.05$. For the proportional fair case, $N_{w}$ is set to 4 and for the high SNR case, $d_{\max }$ is set to $300 \mathrm{~m}$.

Figs. 4.7 show the trajectories of the UAV and mobile nodes for the SDMA and the TDMA scenarios assuming either max-sum or proportional fair objective functions and $\Delta \delta=\frac{\pi}{6}$. The decision-making behavior of the UAV is evident from its ability to appropriately track the nodes as they dynamically change position. Due to the relatively high speed of the UAV, loop maneuvers are necessary to maintain an optimal position for the uplink communications signals. In the proportional-fair approach, the UAV tends to visit the nodes in turn, while the max-sum rate algorithm leads to the UAV approximately tracking the area where the user node density is highest. Note that in this example the proportional-fair algorithm only suffers a slight degradation in overall sum rate compared with the max-sum rate approach.

Figs. 8, 9 show the ergodic sum rate for the different scenarios. For each time step, the rate is calculated by averaging over 1000 independent channel realizations. Results for both $\Delta \delta=\frac{\pi}{6}$ and $\frac{\pi}{9}$ are plotted. Increasing the maximum turning rate will clearly provide better performance since it decreases the extra distance that must be flown to complete a loop maneuver and the amount of time that the array is aligned with the angle-of-arrival of each user's signal (where the ability of the array to suppress interference is minimized). The benefit of using SDMA is also apparent from Figs. 8, 9 , where we see that a rate gain of approximately a factor of 3.3 is achieved over the TDMA scheme. We also note that the obtained sum rate is only about $15 \%$ less than what would be achieved assuming no interference, indicating the effectiveness of the beamforming algorithm.

Fig. 10 compares the average sum rate of the line-search algorithm in (31) with both the low- and high-SNR approximations derived in the previous section. The performance is plotted as a function of the received SNR that would be observed at the UAV from a ground node located at a distance of $1 \mathrm{~km}$. Although the approximate algorithms were derived separately 
under different SNR assumptions, both of them yield performance essentially identical to (31) over all SNR values. Each approximate algorithm is slightly better than the other in its respective SNR regime, but the performance difference is small.

\section{CONCLUSION}

We have investigated the problem of positioning a multiple-antenna UAV for enhanced uplink communications from multiple ground-based users. We studied the optimal UAV trajectory for a case involving two static users, and derived an approximate method for finding this trajectory that only requires a simple line search. For the case of a network of mobile ground users, an adaptive heading algorithm was proposed that uses predictions of the user terminal positions and beamforming at the UAV to maximize SINR at each time step. Two kinds of optimization problems were considered, one that maximizes a lower bound on the average uplink sum rate and one that guarantees fairness among the users using the proportional fair method. Simulation results indicate the effectiveness of the algorithms in automatically generating a suitable UAV heading for the uplink network, and demonstrate the benefit of using SDMA over TDMA in achieving the best throughput performance. We also derived approximate solutions to the UAV heading problem for low- and high-SNR scenarios; the approximations allow for a closed-form solution instead of a line search, but still provide near-optimal performance in their respective domains.

\section{APPENDIX A}

\section{DERIVATION OF UAV TRAJECTORY FOR TWO-USER CASE}

In this appendix, we find an approximation to the problem posed in equation (14):

$$
\begin{array}{cl}
\max _{\delta, C_{a}, C_{b}} & \bar{R} \\
\text { subject } \quad \text { to } & 0 \leq \delta \leq \frac{\pi}{2} \\
& C_{\min } \leq C_{b} \leq C_{a} \leq C_{\max }
\end{array}
$$

where $\bar{R}$ is defined in (13). To begin with, we observe that, due to the symmetric trajectory centered at the midpoint between the two ground nodes, the expected data rate averaged over 
the trajectory will be the same for both users:

$$
\int_{\mathcal{C}} \log _{2}\left(1+S I N R_{1}(p)\right) d p=\int_{\mathcal{C}} \log _{2}\left(1+S I N R_{2}(p)\right) d p .
$$

Thus, we can focus on evaluating the SINR for just one of the users. For large $K$, we can ignore the Rayleigh component of the channel, and assume that $\mathbf{h}_{i}^{\prime} \approx \overline{\mathbf{h}}_{i}$. We replace the explicit dependence of the channel on $n$ with an implicit dependence on a point $p$ along the trajectory defined in Fig. 1. At point $p$, the SINR for user 1 can be expressed as

$$
\begin{aligned}
S I N R_{1} & =\frac{P_{t}}{d_{1}^{\alpha}} \overline{\mathbf{h}}_{1}^{H}\left(\sigma^{2} \mathbf{I}_{M}+\frac{P_{t}}{d_{2}^{\alpha}} \overline{\mathbf{h}}_{2} \overline{\mathbf{h}}_{2}^{H}\right)^{-1} \overline{\mathbf{h}}_{1} \\
& =\frac{M P_{t}}{d_{1}^{\alpha} \sigma^{2}}-\frac{P_{t}^{2}}{d_{1}^{\alpha} d_{2}^{\alpha} \sigma^{4}} \frac{\left|\overline{\mathbf{h}}_{1}^{H} \overline{\mathbf{h}}_{2}\right|^{2}}{1+\frac{M P_{t}}{d_{2}^{\alpha} \sigma^{2}}},
\end{aligned}
$$

where

$$
\left|\overline{\mathbf{h}}_{1}^{H} \overline{\mathbf{h}}_{2}\right|=\left|\frac{\sin \left(\frac{M \pi}{2}\left(\cos \left(\phi_{1}\right) \sin \left(\theta_{1}\right)-\cos \left(\phi_{2}\right) \sin \left(\theta_{2}\right)\right)\right)}{\sin \left(\frac{\pi}{2}\left(\cos \left(\phi_{1}\right) \sin \left(\theta_{1}\right)-\cos \left(\phi_{2}\right) \sin \left(\theta_{2}\right)\right)\right)}\right|,
$$

and $\cos \left(\phi_{i}\right)$ and $\sin \left(\theta_{i}\right)$ are defined in (9). Note that in addition to $\overline{\mathbf{h}}_{1}$, the parameters $d_{i}, \phi_{i}$ and $\theta_{i}$ all implicitly depend on $p$.

Using Jensen's inequality, the following upper bound for $\bar{R}$ can be found:

$$
\bar{R} \leq \log _{2}\left(1+\mathbb{E}\left\{S I N R_{1}\right\}\right)+\log _{2}\left(1+\mathbb{E}\left\{S I N R_{2}\right)\right\}
$$

We will proceed assuming that an operating point that maximizes the upper bound will also approximately optimize $\bar{R}$. Based on (58) and assuming we have a high SNR scenario where $\frac{P_{t}}{d_{i}^{\alpha} \sigma^{2}} \gg 1$

$$
\begin{aligned}
\mathbb{E}\left\{S I N R_{1}\right\} & \stackrel{(d)}{\approx} \frac{P_{t}}{\sigma^{2}} \mathbb{E}\left\{\frac{M}{d_{1}^{\alpha}}-\frac{\left|\mathbf{h}_{1}^{H} \mathbf{h}_{2}\right|^{2}}{d_{1}^{\alpha} M}\right\} \\
& \stackrel{(e)}{\approx} \frac{P_{t}}{d_{1}^{\alpha} \sigma^{2}}\left(M-\frac{\mathbb{E}\left\{\left|\mathbf{h}_{1}^{H} \mathbf{h}_{2}\right|^{2}\right\}}{M}\right),
\end{aligned}
$$

where (d) is due to the high SNR assumption and (e) follows from the assumption that $C_{\max } \ll$ $d_{1}$. The dependence of $S I N R_{1}$ on $d_{2}$ is thus eliminated, and in what follows we drop the subscript on $d_{1}$ and write it simply as $d$. 
Substituting equation (61) in (60), and replacing the objective function in problem (14) with the upper bound of (60), our optimization problem is approximately given by

$$
\begin{aligned}
\max _{\delta, C_{a}, C_{b}} & \log _{2}\left(1+\frac{M P_{t}}{d^{\alpha} \sigma^{2}}-\frac{P_{t} \mathbb{E}\left\{\left|\mathbf{h}_{1}^{H} \mathbf{h}_{2}\right|^{2}\right\}}{M d^{\alpha} \sigma^{2}}\right) \\
\text { subject to } 0 \leq \delta \leq \frac{\pi}{2} & 0 \leq C_{b} \leq C_{a} \leq C_{\max } .
\end{aligned}
$$

Since the objective function in (62) is monotonically decreasing with $\mathbb{E}\left\{\left|\mathbf{h}_{1}^{H} \mathbf{h}_{2}\right|^{2}\right\}$, an equivalent problem is formulated as

$$
\begin{gathered}
\min _{\delta, C_{a}, C_{b}} \mathbb{E}\left\{\left|\mathbf{h}_{1}^{H} \mathbf{h}_{2}\right|^{2}\right\} \\
\text { subject to } 0 \leq \delta \leq \frac{\pi}{2} \\
C_{\min } \leq C_{b} \leq C_{a} \leq C_{\max } .
\end{gathered}
$$

The interpretation of (63) is that the optimal trajectory minimizes the average correlation between the two users' channels.

The calculation of $\mathbb{E}\left\{\left|\mathbf{h}_{1}^{H} \mathbf{h}_{2}\right|^{2}\right\}$ includes the integral of the function

$$
\frac{\sin ^{2}\left(\frac{M \pi}{2}\left(\cos \left(\phi_{1}\right) \sin \left(\theta_{1}\right)-\cos \left(\phi_{2}\right) \sin \left(\theta_{2}\right)\right)\right)}{\sin ^{2}\left(\frac{\pi}{2}\left(\cos \left(\phi_{1}\right) \sin \left(\theta_{1}\right)-\cos \left(\phi_{2}\right) \sin \left(\theta_{2}\right)\right)\right)}
$$

with respect to $p$, which is difficult to evaluate. To simplify (14), we assume that, compared with the distance to the users on the ground, the UAV moves over a small region, and for purposes of analyzing the mathematics, one can assume that the UAV essentially remains fixed at the midpoint between the two users. Only the heading of the UAV changes the uplink rate in this case. Under this assumption, the elevation angles $\phi_{1}, \phi_{2}$ are constant and equal $\phi_{1}=\phi_{2}=\phi^{\prime}$, and the azimuth angles $\theta_{1}, \theta_{2}$ are piecewise constant. When UAV flies along $C_{a}$, they are equal to $\theta_{1}$ and $\theta_{2}$; when the UAV flies along $C_{b}$, they are equal to $\theta_{1}+\frac{\pi}{2}, \theta_{2}+\frac{\pi}{2}$. Note that since $\theta_{2}=\theta_{1}+\pi$ always holds, then $\sin \left(\theta_{2}\right)=-\sin \left(\theta_{1}\right)$ and we have

$$
\left|\mathbf{h}_{1}^{H} \mathbf{h}_{2}\right|^{2}=\frac{\sin ^{2}\left(M \pi \cos \left(\phi^{\prime}\right) \sin \left(\theta_{1}\right)\right)}{\sin ^{2}\left(\pi \cos \left(\phi^{\prime}\right) \sin \left(\theta_{1}\right)\right)}
$$


Note also that $\theta_{1}+\delta=\frac{\pi}{2}$, and hence $\sin \left(\theta_{1}\right)=\cos (\delta)$. Thus

$$
\left|\mathbf{h}_{1}^{H} \mathbf{h}_{2}\right|^{2}=\frac{\sin ^{2}\left(M \pi \cos \left(\phi^{\prime}\right) \cos (\delta)\right)}{\sin ^{2}\left(\pi \cos \left(\phi^{\prime}\right) \cos (\delta)\right)} .
$$

Along $C_{a}$, the UAV flies with heading $\delta$ and along $C_{b}$, the UAV flies with heading $\delta+\frac{\pi}{2}$, so that $\cos \left(\delta+\frac{\pi}{2}\right)=-\sin (\delta)$. Thus, we have

$$
\mathbb{E}\left\{\left|\mathbf{h}_{1}^{H} \mathbf{h}_{2}\right|^{2}\right\}=\frac{C_{a}}{C_{a}+C_{b}} \frac{\sin ^{2}\left(M \pi \cos \left(\phi_{i}^{\prime}\right) \cos (\delta)\right)}{\sin ^{2}\left(\pi \cos \left(\phi_{i}^{\prime}\right) \cos (\delta)\right)}+\frac{C_{b}}{C_{a}+C_{b}} \frac{\sin ^{2}\left(M \pi \cos \left(\phi_{i}^{\prime}\right) \sin (\delta)\right)}{\sin ^{2}\left(\pi \cos \left(\phi_{i}^{\prime}\right) \sin (\delta)\right)} .
$$

Substituting (66) into the objective function of problem (63) yields

$$
\begin{aligned}
& \min _{\delta, C_{a}, C_{b}} \frac{C_{a}}{C_{a}+C_{b}} \frac{\sin ^{2}\left(M \pi \cos \left(\phi_{i}^{\prime}\right) \cos (\delta)\right)}{\sin ^{2}\left(\pi \cos \left(\phi_{i}^{\prime}\right) \cos (\delta)\right)}+\frac{C_{b}}{C_{a}+C_{b}} \frac{\sin ^{2}\left(M \pi \cos \left(\phi_{i}^{\prime}\right) \sin (\delta)\right)}{\sin ^{2}\left(\pi \cos \left(\phi_{i}^{\prime}\right) \sin (\delta)\right)} \\
& \text { subject to } \quad 0 \leq \delta \leq \frac{\pi}{2} \\
& C_{\min } \leq C_{b} \leq C_{a} \leq C_{\max } .
\end{aligned}
$$

We now show that Problem (67) is equivalent to an optimization problem over the single variable $\delta$. First define

$$
\begin{aligned}
s_{1} & =\frac{\sin ^{2}\left(M \pi \cos \left(\phi_{i}^{\prime}\right) \cos (\delta)\right)}{\sin ^{2}\left(\pi \cos \left(\phi_{i}^{\prime}\right) \cos (\delta)\right)} \\
s_{2} & =\frac{\sin ^{2}\left(M \pi \cos \left(\phi_{i}^{\prime}\right) \sin (\delta)\right)}{\sin ^{2}\left(\pi \cos \left(\phi_{i}^{\prime}\right) \sin (\delta)\right)} \\
R_{c} & =\frac{C_{\max }}{C_{\min }} \\
R & =\frac{C_{a}}{C_{b}},
\end{aligned}
$$

so that $1 \leq R \leq R_{c}$. Then the objective function of (67) can be rewritten as

$$
\frac{R}{1+R} s_{1}+\frac{1}{1+R} s_{2}=s_{1}+\frac{s_{2}-s_{1}}{1+R} .
$$

Given a heading direction $\delta \in\left[0, \frac{\pi}{2}\right]$, if $s_{2} \geq s_{1}$, then the objective function is minimized when $R=R_{c}$. Otherwise, if $s_{2}<s_{1}, R=1$ minimizes the objective function. The domain $\left[0, \frac{\pi}{2}\right]$ can be divided into two sets $\mathcal{S}_{1}$ and $\mathcal{S}_{2}$, such that for $\delta \in \mathcal{S}_{1}, s_{2}<s_{1}$, and for $\delta \in \mathcal{S}_{2}, s_{2} \geq s_{1}$. Then problem (67) can be divided into two subproblems

$$
\min _{\delta} \frac{R_{c}}{1+R_{c}} s_{1}+\frac{1}{1+R_{c}} s_{2}
$$

subject to $\delta \in \mathcal{S}_{2}$. 


$$
\min _{\delta} \frac{1}{2} s_{2}+\frac{1}{2} s_{1}
$$

subject to $\delta \in \mathcal{S}_{1}$.

Since $s_{1}\left(\frac{\pi}{2}-\delta\right)=s_{2}(\delta)$, for each $\delta \in \mathcal{S}_{2}$, we have $\frac{\pi}{2}-\delta \in \mathcal{S}_{1}$ and vice versa. Thus the following equation holds

$$
\frac{R_{c}}{1+R_{c}} s_{1}(\delta)+\frac{1}{1+R_{c}} s_{2}(\delta)<\frac{1}{2} s_{1}(\delta)+\frac{1}{2} s_{2}(\delta)=\frac{1}{2} s_{2}\left(\frac{\pi}{2}-\delta\right)+\frac{1}{2} s_{1}\left(\frac{\pi}{2}-\delta\right) .
$$

Then the minimum value of (73) must be smaller than the minimum value of (74) and problem (67) is equivalent to problem (73). For each $\delta \in \mathcal{S}_{2}$, the following equation holds

$$
\frac{R_{c}}{1+R_{c}} s_{1}(\delta)+\frac{1}{1+R_{c}} s_{2}(\delta)<\frac{R_{c}}{1+R_{c}} s_{2}(\delta)+\frac{1}{1+R_{c}} s_{1}(\delta)=\frac{R_{c}}{1+R_{c}} s_{1}\left(\frac{\pi}{2}-\delta\right)+\frac{1}{1+R_{c}} s_{2}\left(\frac{\pi}{2}-\delta\right)
$$

and problem (73) is thus equivalent to

$$
\begin{aligned}
\min _{\delta} & \frac{R_{c}}{1+R_{c}} s_{1}+\frac{1}{1+R_{c}} s_{2} \\
\text { subject to } & 0<\delta<\frac{\pi}{2} .
\end{aligned}
$$

Equation (15) follows directly from (77).

\section{REFERENCES}

[1] C. Cheng, P. Hsiao, H. Kung, and D. Vlah, "Maximizing throughtput of UAV-relaying networks with the load-carry-anddeliver paradigm," in Proc. IEEE WCNC 2007, Mar. 2007, pp. 4417-4424.

[2] Z. Han, A. L. Swindlehurst, and K. J. R. Liu, "Optimization of MANET connectivity via smart deployment/movement of unmanned air vehicles," IEEE Trans. Veh. Technol., vol. 58, no. 7, pp. 3533-3546, Sep. 2009.

[3] E. P. de Freitas, T. Heimfarth, I. F. Netto, C. E. Lino, C. E. Pereira, A. M. Ferreira, F. R. Wagner, and T. Larsson, "UAV relay network to support WSN connectivity,” in Proc. IEEE ICUMT 2010, Oct. 2010, pp. 309-314.

[4] R. Palat, A. Annamalau, and J. Reed, "Cooperative relaying for ad-hoc ground networks using swarm UAVs," in Proc. IEEE MILCOM 2005, Oct. 2005, pp. 1588-1594.

[5] P. Zhan, K. Yu, and A. L. Swindlehurst, "Wireless relay communications with unmannned aerial vehicles: performance and optimization," IEEE Trans. Aero. and Elec. Sys. (to appear), 2010.

[6] I. Rubin and R. Zhang, "Placement of UAVs as communication relays aiding mobile ad hoc wireless networks," in Proc. IEEE MILCOM 2007, Oct. 2007, pp. 1-7.

[7] J. L. Hillman, S. D. Jones, R. A. Nichols, and I. J. Wang, "Communications network architectures for the army future combat system and objective force," in Proc. IEEE MILCOM 2002, Oct. 2002, pp. 1417-11 421.

[8] M. F. J. Pinkney, D. Hampel, and S. DiPierro, "Unmanned aerial vehicle (UAV) communications relay," in Proc. IEEE MILCOM 1996, Oct. 1996, pp. 47-51. 
[9] M. S. Sharawi, D. Aloi, and O. A. Rawashdeh, "Design and implementation of embedded printed antenna arrays in small UAV wing structures," IEEE Trans. Antennas and Propag., vol. 58, no. 8, pp. 2531-2538, Aug. 2010.

[10] H. T. Kung, C.-K. Lin, T.-H. Lin, S. J. Tarsa, and D. Vlah, "Measuring diversity on a low-altitude UAV in a ground-to-air wireless 802.11 mesh network," in Proc. IEEE Globecom Workshop on Wireless Networking for Unmanned Aerial Vehicles, Dec. 2010, pp. 1799-1804.

[11] F. Jiang and A. L. Swindlehurst, "Dynamic UAV relay positioning for the ground-to-air uplink," in Proc. IEEE Globecom Workshop on Wireless Networking for Unmanned Aerial Vehicles, Dec. 2010, pp. 1766-1770.

[12] J. H. Winters, "Optimum combining in digital mobile radio with cochannel interference," IEEE Trans. Veh. Technol., vol. VT-33, no. 3, pp. 144-155, Aug. 1984.

[13] H. Bolcskei, M. Borgmann, and A. J. Paulraj, "Impact of the propagation environment on the performance of spacefrequency coded MIMO-OFDM,” IEEE J. Sel. Areas Commun., vol. 21, no. 3, pp. 427-439, Apr. 2003.

[14] K. Varma, "Time-delay-estimate based direction-of-arrival estimation for speech in reverberant environments," Master's thesis, Virginia Polytechnic Institute and State University, 2002.

[15] T. Trump and B. Ottersten, "Estimation of nominal direction of arrival and angular spread using an array of sensors," Signal Processing, vol. 50, no. 1-2, pp. 57-69, Apr. 1996.

[16] Z. Zaidi, B. Mark, and R. Thomas, "A two-tier representation of node mobility in ad hoc networks," in Proc. IEEE SECON 2004, Oct. 2004, pp. 153-161.

[17] X. Zhang, D. P. Palomar, and B. Ottersten, "Statistically robust design for linear MIMO transceivers," IEEE Trans. Signal Process., vol. 56, no. 8, pp. 3678-3689, Aug. 2008.

[18] J. Holtzman, "Asymptotic analysis of proportional fair algorithm," in Proc. IEEE PIMRC 2001, Sep. 2001 , pp. F33-F37.

[19] G. W. Stewart, Matrix Algorithms Volume I: Basic Decompositions. Philadelphia: SIAM, 1998. 


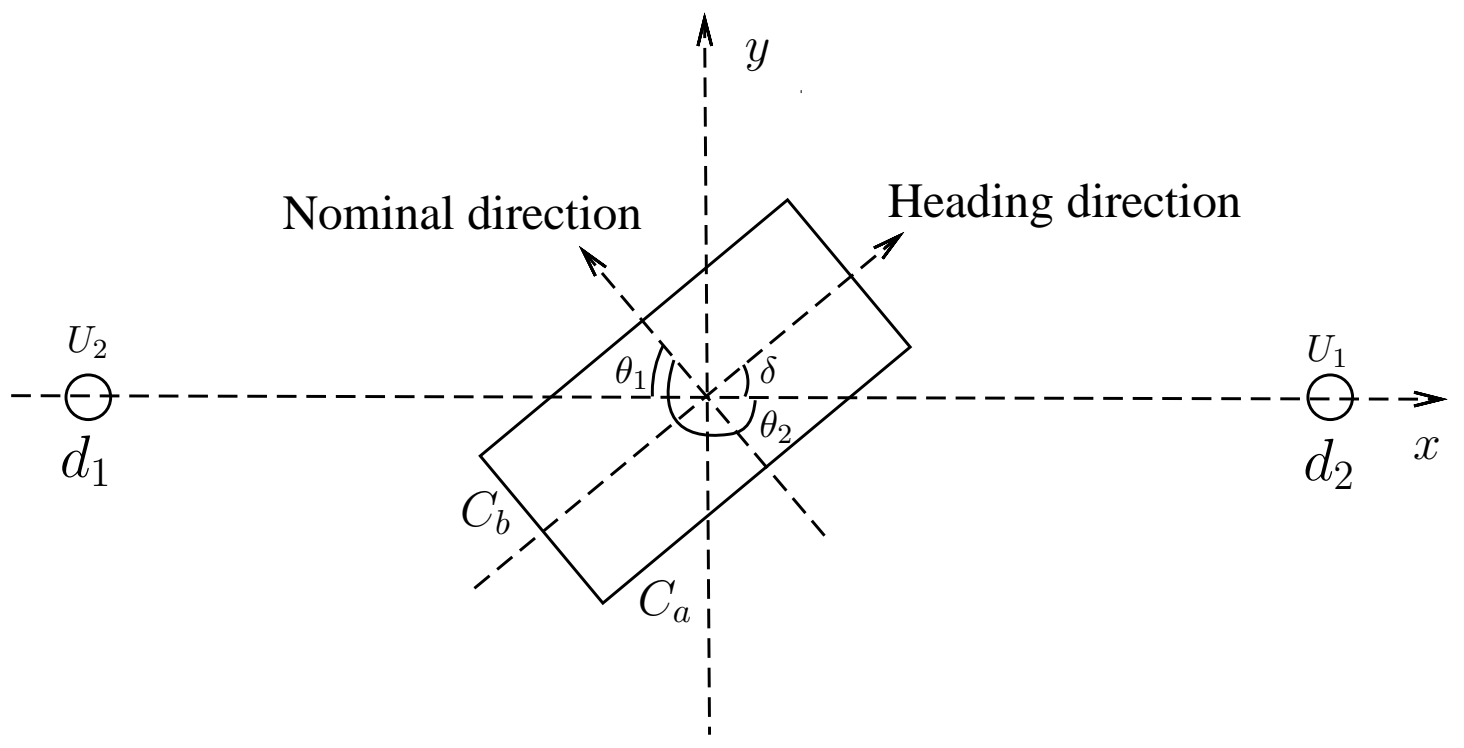

Fig. 1. Illustration of the rectangular UAV trajectory with edge lengths $C_{a}$ and $C_{b}$. The angles $\theta_{1}$ and $\theta_{2}$ are determined when the UAV is at the midpoint of the two users with heading direction $\delta$.

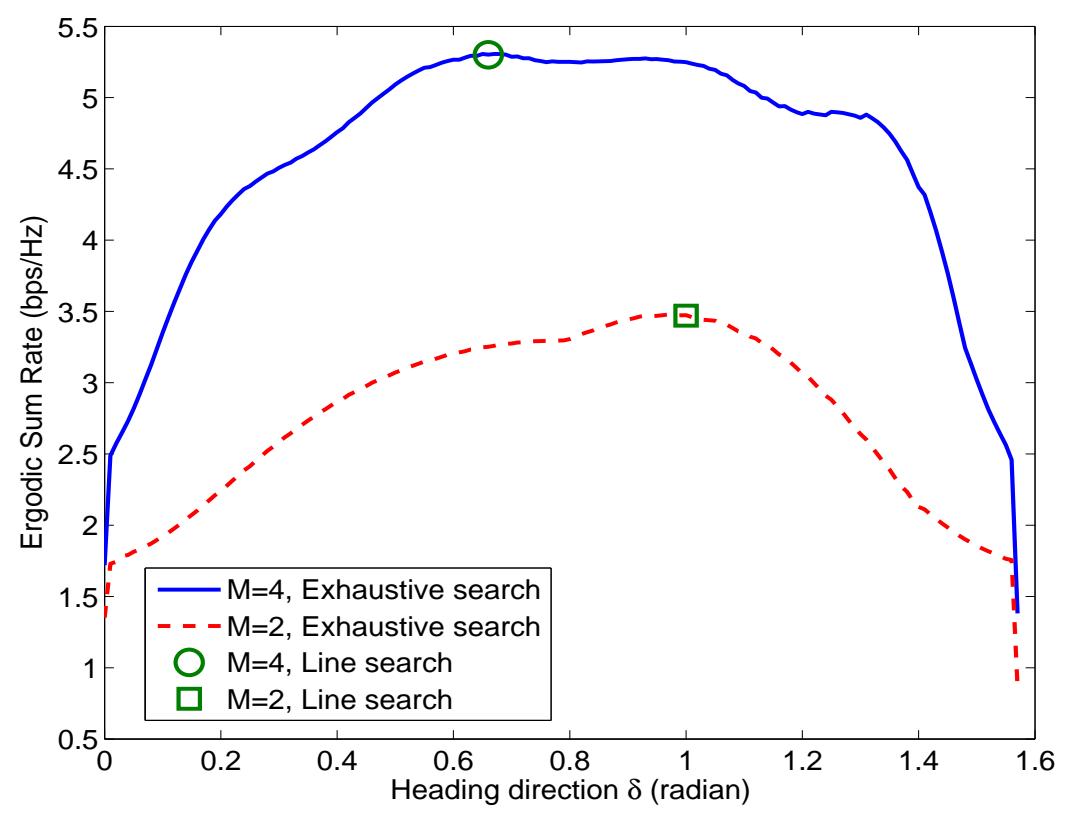

Fig. 2. Orientation of the rectangular trajectory provided by the line search method in 15]. For the exhaustive search method, the solid curve and the dashed curve denote the optimal sum rate that can be achieved for different orientations $\delta$. When $M=4$, the optimal $\delta$ are: 0.66 (exhaustive search), 0.69 (line search); when $M=2$, the optimal $\delta$ are: 0.98 (exhaustive search), 1.00 (line search). 


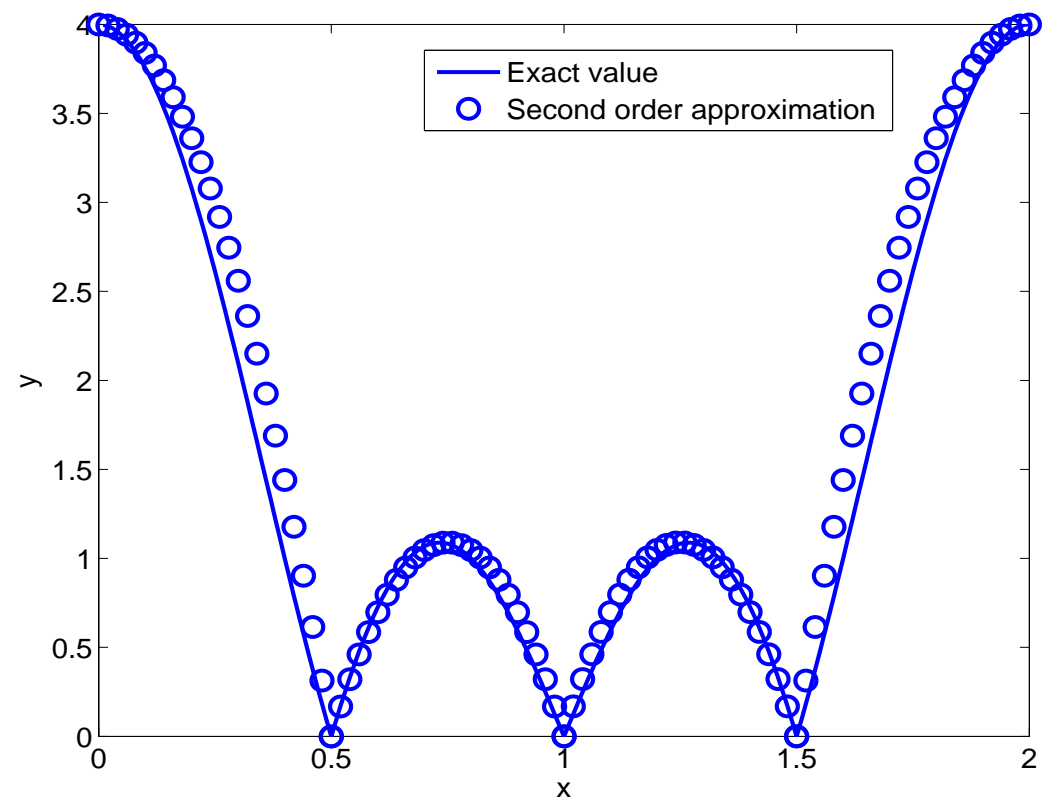

Fig. 3. Plot of $\left|\overline{\mathbf{h}}_{i}^{H} \overline{\mathbf{h}}_{j}\right|$ as a function of the AoA between the two users, along with a set of piecewise quadratic approximations.

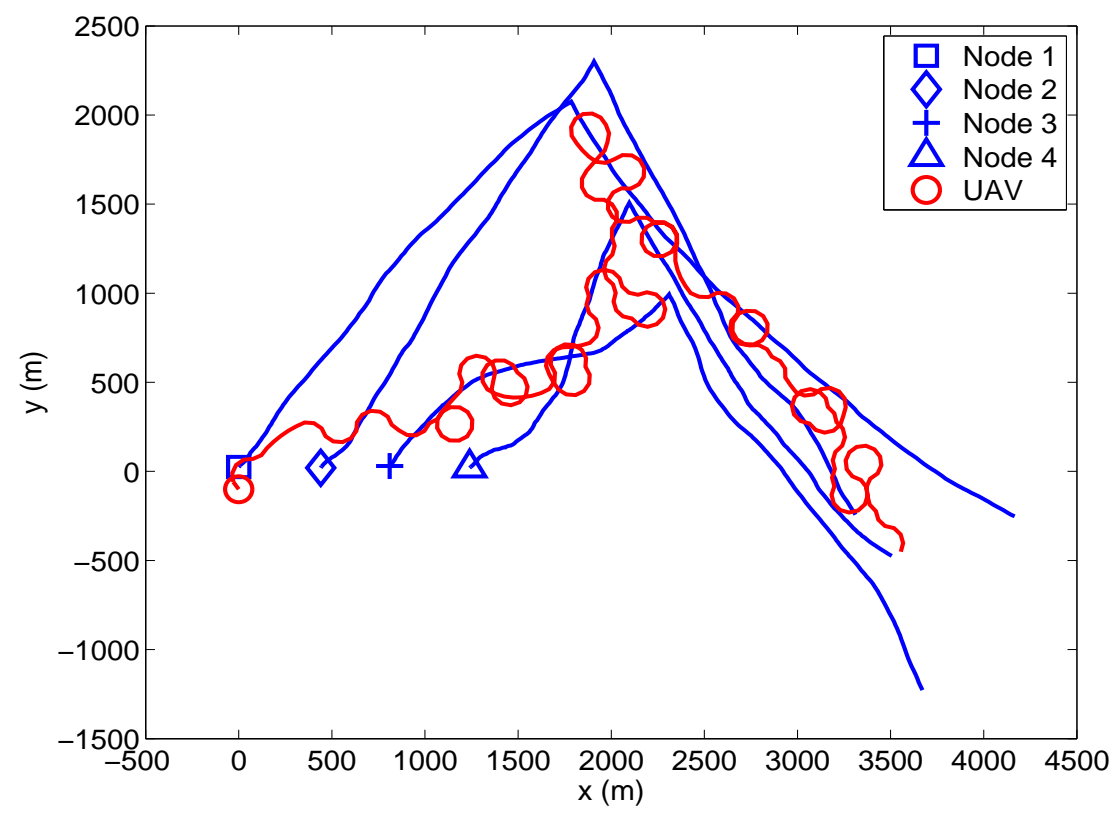

Fig. 4. Trajectories of the UAV and user nodes for SDMA with $\Delta \delta=\frac{\pi}{6}, K=10$ and $\frac{P_{t}}{\sigma^{2}}=45 \mathrm{~dB}$, maximizing sum rate. The average sum rate is: $1.8185 \mathrm{bps} / \mathrm{Hz}$. The single user data rates are $\mathrm{u}_{1}=0.5607, \mathrm{u}_{2}=0.6138, \mathrm{u}_{3}=0.2406, \mathrm{u}_{4}=0.4034$. 


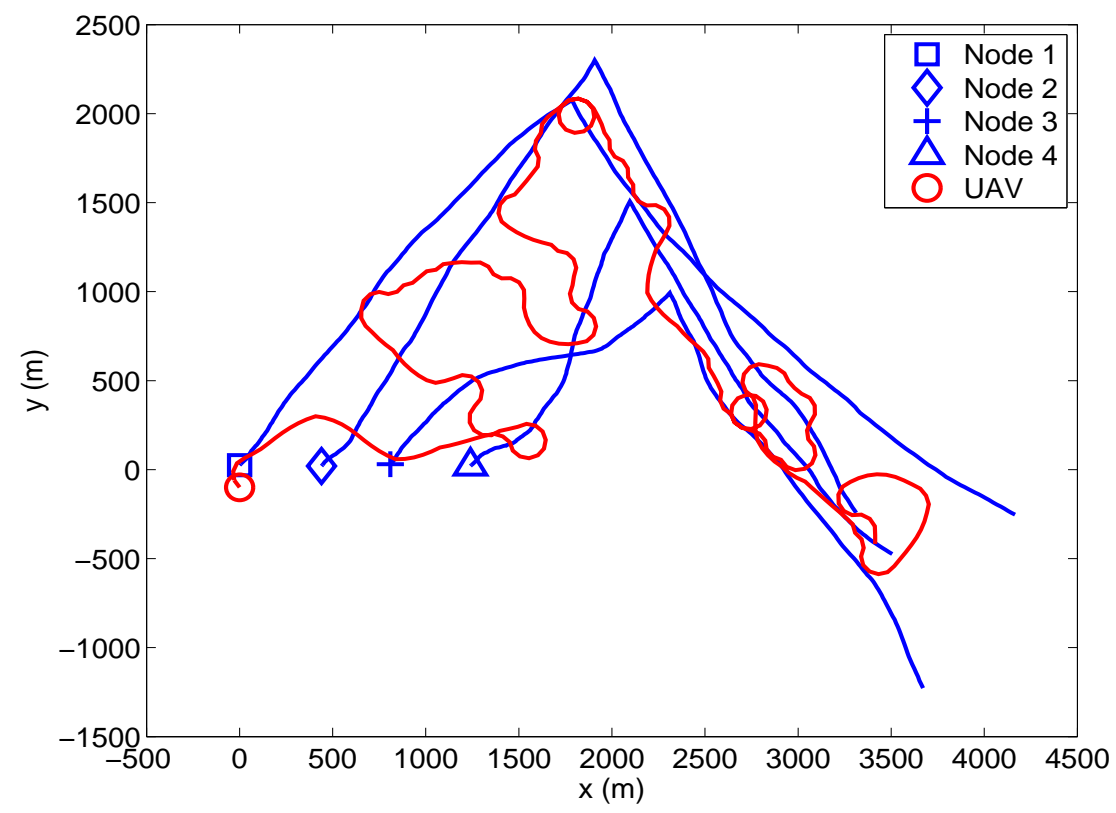

Fig. 5. Trajectories of the UAV and user nodes for SDMA with $\Delta \delta=\frac{\pi}{6}, K=10$ and $\frac{P_{t}}{\sigma^{2}}=45 \mathrm{~dB}$, proportional fair. The average sum rate is $1.6968 \mathrm{bps} / \mathrm{Hz}\left(\mathrm{u}_{1}=0.4169, \mathrm{u}_{2}=0.4084, \mathrm{u}_{3}=0.4088, \mathrm{u}_{4}=0.4627\right)$.

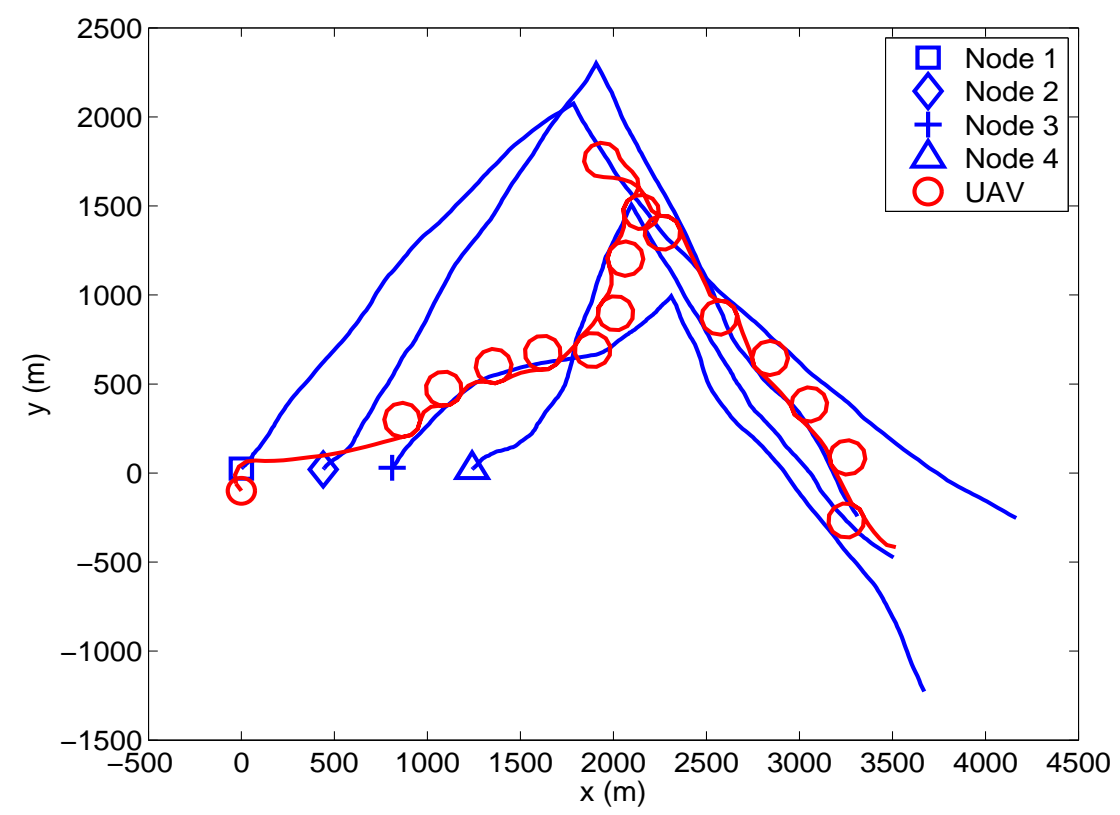

Fig. 6. Trajectories of the UAV and user nodes for TDMA with $\Delta \delta=\frac{\pi}{6}, K=10$ and $\frac{P_{t}}{\sigma^{2}}=45 \mathrm{~dB}$, maximizing sum rate. The average sum rate is: $0.5294 \mathrm{bps} / \mathrm{Hz}\left(\mathrm{u}_{1}=0.1418, \mathrm{u}_{2}=0.1674, \mathrm{u}_{3}=0.0895, \mathrm{u}_{4}=0.1307\right)$. 


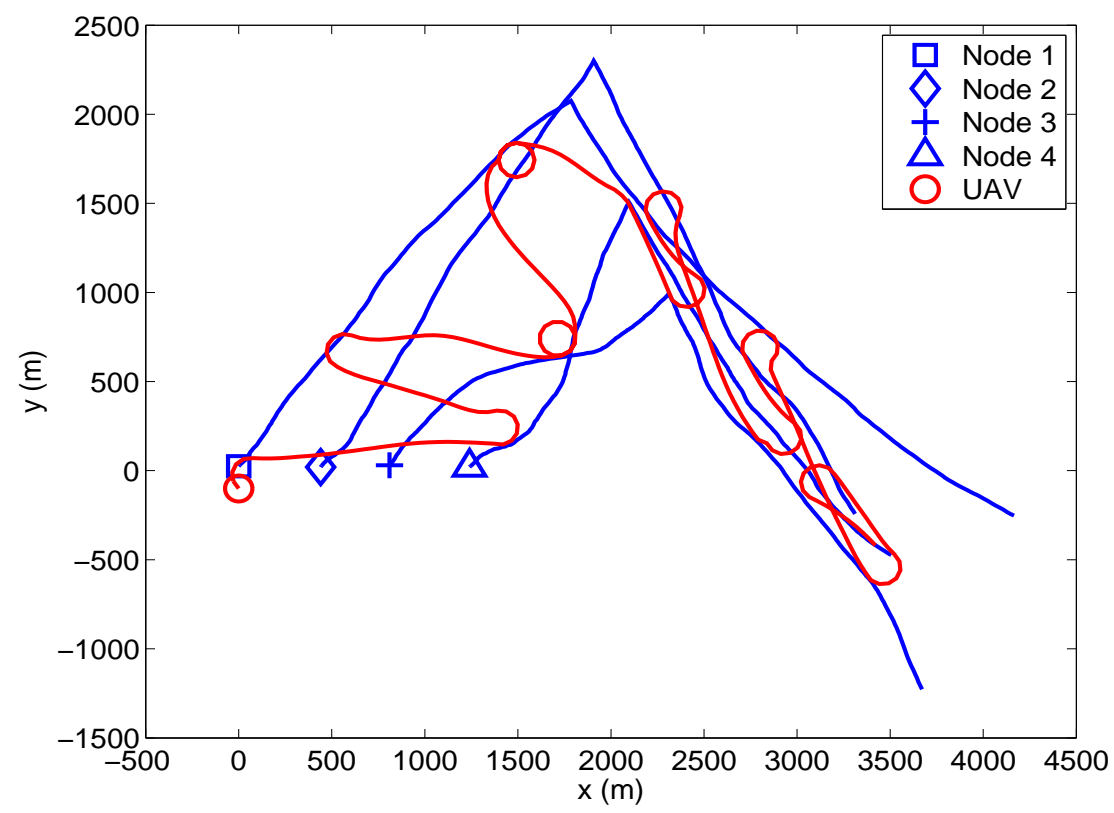

Fig. 7. Trajectories of the UAV and user nodes for TDMA with $\Delta \delta=\frac{\pi}{6}, K=10$ and $\frac{P_{t}}{\sigma^{2}}=45 \mathrm{~dB}$, proportional fair. The average sum rate is: $0.5139 \mathrm{bps} / \mathrm{Hz}\left(\mathrm{u}_{1}=0.1222, \mathrm{u}_{2}=0.1274, \mathrm{u}_{3}=0.1193, \mathrm{u}_{4}=0.1450\right)$.

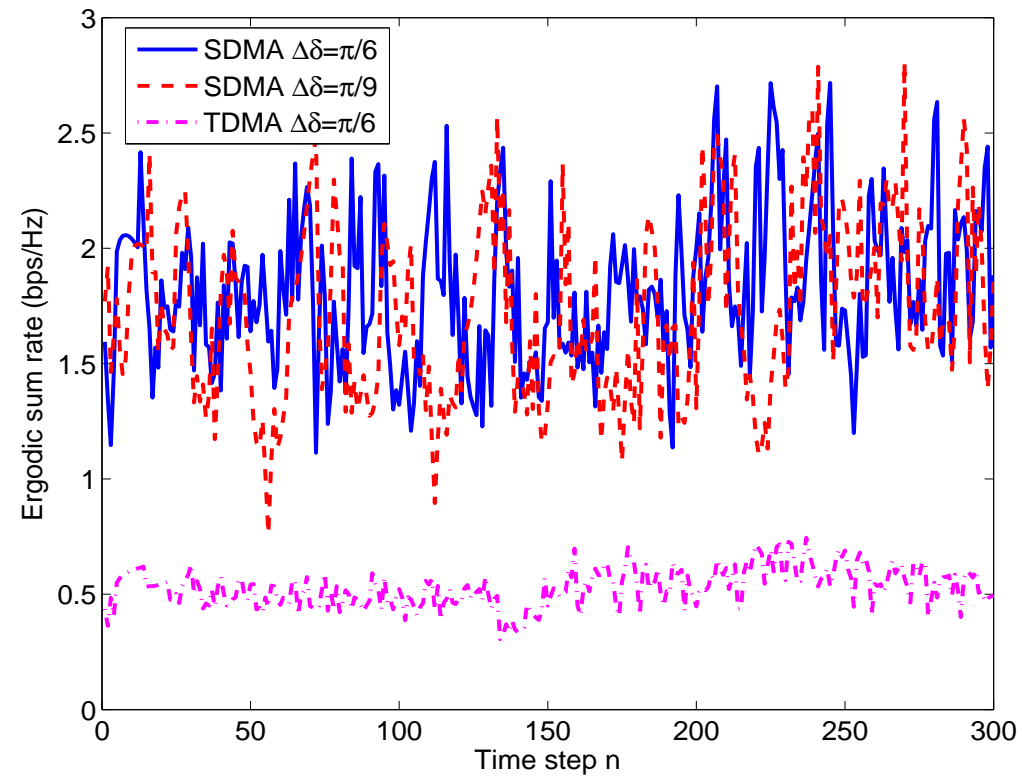

Fig. 8. Comparison of sum rate performance (bps/Hz) with $K=10$ and $\frac{P_{t}}{\sigma^{2}}=45 \mathrm{~dB}$, maximizing sum rate. The average sum rates are: $1.8185\left(\mathrm{SDMA}, \Delta \delta=\frac{\pi}{6}\right), 1.7247\left(\mathrm{SDMA}, \Delta \delta=\frac{\pi}{9}\right), 0.5294\left(\mathrm{TDMA}, \Delta \delta=\frac{\pi}{6}\right)$. 


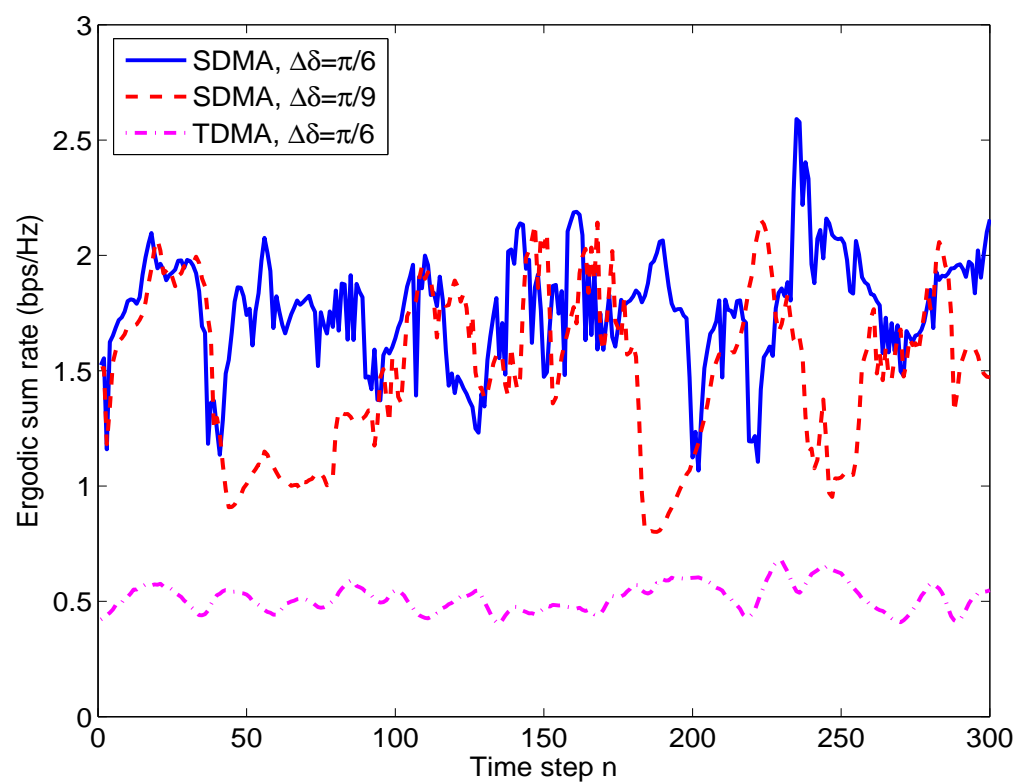

Fig. 9. Comparison of sum rate performance (bps/Hz) with $K=10$ and $\frac{P_{t}}{\sigma^{2}}=45 \mathrm{~dB}$, proportional fair. The average sum rates are: $1.6968\left(\mathrm{SDMA}, \Delta \delta=\frac{\pi}{6}\right), 1.6042\left(\mathrm{SDMA}, \Delta \delta=\frac{\pi}{9}\right), 0.5139\left(\mathrm{TDMA}, \Delta \delta=\frac{\pi}{6}\right)$.

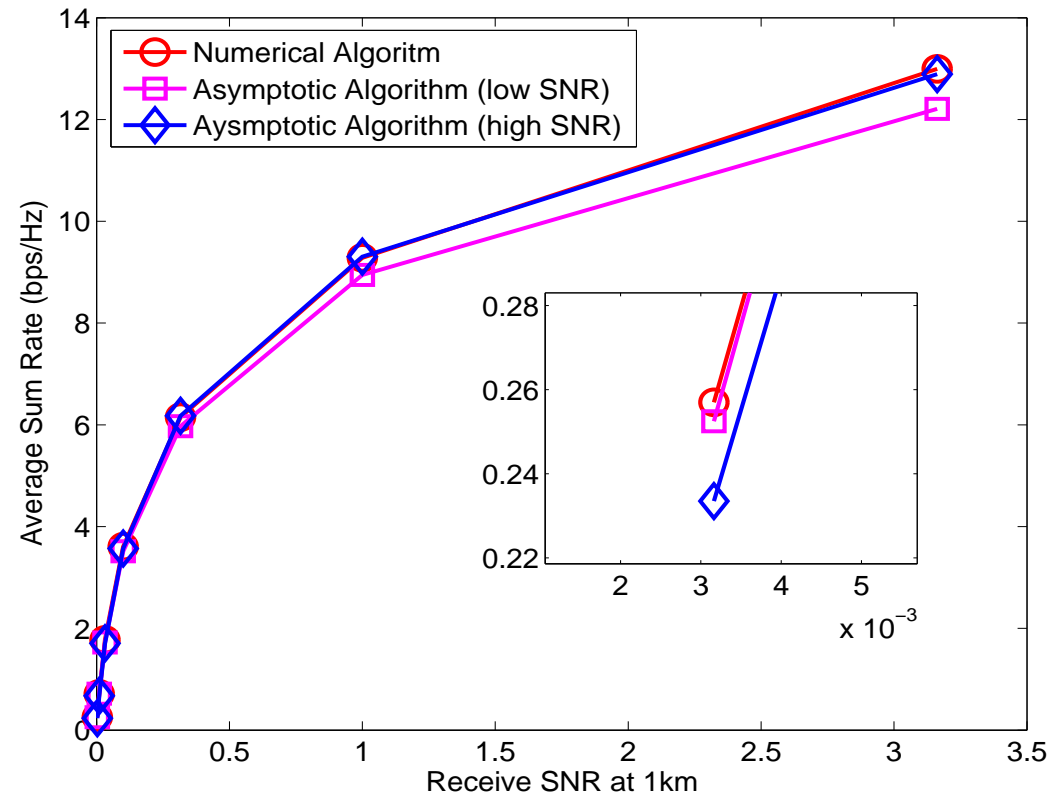

Fig. 10. Comparison of the average sum rate of the line-search and closed-form approximations with $\Delta \delta=\frac{\pi}{9}, K=1000$, maximizing sum rate. The $\mathrm{x}$-axis denotes the SNR that would be observed at the UAV for a user node at a distance of $1 \mathrm{~km}$. 